\title{
Synthetic studies on taxanes: A domino-enyne metathesis/Diels-Alder approach to the $\mathrm{AB}$-ring
}

\author{
KRISHNA P KALIAPPAN*, VELAYUTHAM RAVIKUMAR and SANDIP A PUJARI \\ Department of Chemistry, Indian Institute of Technology Bombay, Mumbai 400076 \\ e-mail: kpk@iitb.ac.in
}

\begin{abstract}
A domino enyne cross-metathesis/intramolecular Diels-Alder reaction has been successfully used to synthesize a bicyclo[5.3.1] undecene, corresponding to AB-ring of taxol without the gem dimethyl group.
\end{abstract}

Keywords. Taxol; RCM; Grubbs catalyst; IMDA; Anticancer; Domino reaction.

\section{Introduction}

Taxol ${ }^{\circledR}$ (Paclitaxel: 1), a remarkable cytotoxic diterpene having a complex molecular architecture, was first isolated from the bark of the Pacific yew tree, Taxus brevifolia, in 1967 by Wall and Wani, and the structure was elucidated in 1971 by the combination of X-ray studies and ${ }^{1} \mathrm{H}$ NMR analysis. ${ }^{1}$ It was identified as the first member of the novel group of anticancer agents (figure 1), which promotes the assembly of the proteins $\alpha$ - and $\beta$-tubulin into microtubules and disturbs the polymerization-depolymerization dynamics by making the microtubules extremely stable. This makes the cell division impossible, resulting in cell death. This novel mechanism of taxol was in contrast to the existing anticancer drugs, viz. colchicine, podophyllotoxin and dolastatins, which bind to free tubulin and interrupt the growth of microtubules. ${ }^{2}$ In view of the importance of the biological activity and scarce availability, many groups actively pursued strategies that aimed at the chemical synthesis of taxol. The potential problems anticipated in the synthesis are: (a) construction of a highly distorted and functionalized ABC-tricarbocyclic structure and (b) control of stereochemistry at highly congested asymmetric centers. Regardless of these problems, six synthetic groups have achieved the total synthesis of taxol using a variety of approaches. ${ }^{3-8}$

These syntheses were landmarks in the field of organic synthesis and the approaches for the construction of the basic skeleton of taxol could be divided into three types: (a) elaboration of naturally occur ring terpenes to the $\mathrm{AB}$ ring system of taxol by epoxy-

\footnotetext{
*For correspondence
}

alcohol fragmentation, e.g. total synthesis by Holton et $a l^{3}$ and by Wender et al. ${ }^{6}$ (b) convergent strategies including a B-ring closure reaction of connected A-C ring system, e.g. total synthesis by Nicolaou et $a l,{ }^{4}$ Danishefsky et $a l^{5}$ and the Kuwajima group ${ }^{7}$ and (c) a unique pathway to taxol core, starting with an acyclic precursor to form B-ring, executed by the Mukaiyama group. ${ }^{8}$

Unfortunately, even the shortest synthesis ${ }^{6}$ of taxol known to date involves 37 steps with an overall yield of approximately $0.4 \%$, which made the chemical synthesis of taxol less impressive on industrial scale. Gratifyingly, the semi synthesis of taxol from 10deacetylbaccatin III (10-DAB) solved its supply problem as 10-DAB could be easily isolated from the leaves and twigs of the European yew, Taxus baccata, at approximately $0 \cdot 1 \%$ by dry weight, without creating any environment hazards. ${ }^{9}$ The relative ease in accessing the taxol through semi synthesis subdued the impact of total synthesis of taxol on its supply problems. On the other hand, synthesizing the analogues of taxol with less molecular complexity and comparable cytotoxicity to the parent molecule is an attractive target and also those analogues which are not available from natural sources but derived through chemical synthesis could be of biological interest.

\section{Experimental}

\subsection{General}

Unless and otherwise noted, all starting materials and reagents were obtained from commercial supp- 
liers and used after further purification. Tetrahydrofuran was distilled from sodium benzophenone ketyl and toluene from sodium. N,N- dimethyl formamide was distilled from $\mathrm{MgSO}_{4}$. Dichloromethane, hexane and pyridine were freshly distilled from calcium hydride. All solvents for routine isolation of products and chromatography were reagent grade and glass distilled. Reaction flasks were dried in oven at $100^{\circ} \mathrm{C}$ for $12 \mathrm{~h}$. Air and moisture sensitive reactions were performed under an argon/UHP nitrogen atmosphere. Flash chromatography was performed using silica gel (100-200 mesh, Aceme) with indicated solvents. All reactions were monitored by thin-layer chromatography carried out on $0.25 \mathrm{~mm}$ E. Merck silica plates $(60 \mathrm{~F}-254)$ using UV light as visualizing agent and $7 \%$ ethanolic phosphomolybdic acid and heat as developing agents. Optical rotation was recorded on Jasco DIP-370 or Autopol IV digital polarimeter. IR spectra were recorded from Thermo Nicolet Avater 320 FT-IR and Nicolete Impact 400 machine. Mass spectra were obtained with Waters Micromass-QTof micro ${ }^{\mathrm{TM}}$ (YA105) spectrometer. ${ }^{1} \mathrm{H}$ and ${ }^{13} \mathrm{C}$ NMR spectra were recorded either on Varian AS 400 or Varian ASM 300. Values are listed as chemical shift, multiplicity $(s$, singlet; $d$, doublet; $t$, triplet; $q$, quartet; $m$, multiplet; $d d$, doublet of doublet; $b s$, broad singlet), number of protons and coupling constant in hertz $(\mathrm{Hz})$.

2.1a (4S, 5S, 6S)-1-(6-Methoxy-2,2-dimethyl-6-vinyltetrahydrofuro[3,4-d][1,3]dioxol-4yl)-ethane-(S)-1,2diol (10): To a solution of mannose diacetonide 9 $(3.12 \mathrm{~g}, 12 \mathrm{mmol})$ in dry THF $(72 \mathrm{~mL})$, at $0^{\circ} \mathrm{C}$ was added vinylmagnesium bromide $(1.0 \mathrm{M}$ in THF, $36 \mathrm{~mL}, 36 \mathrm{mmol}$ ) drop-wise. After stirring at $0^{\circ} \mathrm{C}$ for $2 \mathrm{~h}$, the reaction mixture was allowed to attain room temperature and stirred for $12 \mathrm{~h}$. The reaction was quenched with saturated aqueous ammonium chloride $(50 \mathrm{~mL})$ and extracted with ethyl acetate $(3 \times 50 \mathrm{~mL})$. The combined organic extracts were dried over anhydrous sodium sulphate and evaporated under reduced pressure to give a residue which was purified by silica gel chromatography $(40 \%$, EtOAc/ hexanes) to afford a mixture of diol $(3 \cdot 1 \mathrm{~g}, 90 \%)$ and traces of its epimer as colourless oils which solidified on storage at $0{ }^{\circ} \mathrm{C} . R_{f}=0.6(2: 3$ ethyl acetate/ hexanes); IR (neat) 3405, 3098, 1643, $1068 \mathrm{~cm}^{-1} ;{ }^{1} \mathrm{H}$ NMR (300 MHz, $\left.\mathrm{CDCl}_{3}\right): \delta 5.97(d d d, 1 \mathrm{H}, J=17 \cdot 7$, $10 \cdot 2,5 \cdot 1 \mathrm{~Hz},=\mathrm{C} \underline{\mathrm{H}}), 5 \cdot 47-5.25\left(m, 2 \mathrm{H}, \mathrm{C}_{2}=\right), 4.79$ $(d d, \quad 1 \mathrm{H}, \quad J=6.0,3.6 \mathrm{~Hz}, \mathrm{C} \underline{\mathrm{H}}), 4.58(d, 1 \mathrm{H}$, $J=6.6 \mathrm{~Hz}, \mathrm{C} \underline{\mathrm{H}}), 4.42-4.32\left(m, 2 \mathrm{H}, \mathrm{C}_{2}\right), 4.18-$ $4.00\left(m, 3 \mathrm{H}, \mathrm{C} \underline{\mathrm{H}}\right.$ and $\left.\mathrm{CH}_{2}\right), 3 \cdot 89-3.85(m, 1 \mathrm{H}, \mathrm{C} \underline{\mathrm{H}})$, $3.49(d, 1 \mathrm{H}, J=6.6 \mathrm{~Hz}, \mathrm{C} \underline{\mathrm{H}}), 1.52\left(s, 3 \mathrm{H}, \mathrm{C}_{3}\right)$, $1.39\left(s, 3 \mathrm{H}, \underline{\mathrm{CH}}_{3}\right), 1.37\left(s, 3 \mathrm{H}, \mathrm{C}_{3}\right), 1.34(s, 3 \mathrm{H}$, $\left.\mathrm{C}_{3}\right) ;{ }^{13} \mathrm{C}$ NMR $\left(100 \mathrm{MHz}, \mathrm{CDCl}_{3}\right): \delta 133 \cdot 7,118 \cdot 6$, $111 \cdot 7,104 \cdot 7,85 \cdot 3,80 \cdot 9,75 \cdot 4,70 \cdot 0,37 \cdot 8,26 \cdot 8,26 \cdot 2$.

To a stirred suspension of $\mathrm{MnO}_{2} \quad(15.1 \mathrm{~g}$, $173.6 \mathrm{mmol}$ ) in $100 \mathrm{~mL}$ of dry $\mathrm{CH}_{2} \mathrm{Cl}_{2}$, a solution of above diol $(2.5 \mathrm{~g}, 8.68 \mathrm{mmol})$, in $10 \mathrm{~mL}$ of $\mathrm{CH}_{2} \mathrm{Cl}_{2}$ was added drop-wise and stirred for $12 \mathrm{~h}$ at room temperature. TLC of the reaction was monitored until disappearance of the starting material. The mixture was filtered through a sintered glass funnel, concentrated in vacuo and purified by silica gel chromatography (33\%, EtOAc/hexanes) to afford a lactol $(2 \cdot 3 \mathrm{~g}$, $92 \%)$ as a colourless oil. $R_{f}=0.6(1: 4$ ethyl acetate/ hexanes); $[\alpha]_{D}^{25}=+35.8$ (c 1.7, $\mathrm{CHCl}_{3}$ ); IR (neat) $3409,3104,1737,1637 \mathrm{~cm}^{-1}$; ${ }^{1} \mathrm{H}$ NMR $(300 \mathrm{MHz}$, $\left.\mathrm{CDCl}_{3}\right): \delta 6.06(d d, 1 \mathrm{H}, J=17 \cdot 7,11 \cdot 1 \mathrm{~Hz}, \mathrm{C} \underline{\mathrm{H}}=)$, 5.63-5.57 ( $m, 1 \mathrm{H}, \mathrm{C} \underline{\mathrm{H}}=), 5.42-5.38(m, 1 \mathrm{H}, \mathrm{C} \underline{\mathrm{H}}=)$, $4.92(d d, 1 \mathrm{H}, J=6,3.6 \mathrm{~Hz}, \mathrm{CH}), 4.50(d, 1 \mathrm{H}$, $J=5.7 \mathrm{~Hz}, \mathrm{CH}), 4.51-4.44(m, 1 \mathrm{H}, \mathrm{CH}), 4.21(\mathrm{dd}$, $1 \mathrm{H}, J=7.5,3.6 \mathrm{~Hz}, \mathrm{C} \underline{\mathrm{H}}), 4.18-4.05\left(m, 2 \mathrm{H}, \mathrm{CH}_{2}\right)$, $3.16(b r s, 1 \mathrm{H}, \mathrm{OH}), 1.52\left(s, 3 \mathrm{H}, \mathrm{CH}_{3}\right) 1.50(s, 3 \mathrm{H}$, $\left.\mathrm{CH}_{3}\right), 1.42\left(s, 3 \mathrm{H}, \mathrm{CH}_{3}\right), 1.36\left(s, 3 \mathrm{H}, \mathrm{CH}_{3}\right) ;{ }^{13} \mathrm{C}$ NMR $\left(100 \mathrm{MHz}, \mathrm{CDCl}_{3}\right): \delta 136 \cdot 3,117 \cdot 4,112 \cdot 8$, $109 \cdot 1,104 \cdot 4,86 \cdot 7,80 \cdot 3,79 \cdot 2,73 \cdot 3,66 \cdot 6,26 \cdot 8,25 \cdot 8$, 25.2, 24.4; LRMS (ES) $[\mathrm{M}+\mathrm{Na}]^{+}$309.1693; HRMS (ES) calcd. for $\mathrm{C}_{14} \mathrm{H}_{22} \mathrm{O}_{6} \mathrm{Na} \mathrm{m} / z$ 309.1314, found $\mathrm{m} / \mathrm{z}$ $309 \cdot 1315$.

To a stirred solution of the above lactol $(650 \mathrm{mg}$, $2.27 \mathrm{mmol})$, in dry methanol $(20 \mathrm{~mL})$ was added PPTS $(1.14 \mathrm{~g}, 4.5 \mathrm{mmol})$ and the mixture was stirred for $12 \mathrm{~h}$ at room temperature. The reaction was quenched with saturated aqueous sodium bicarbonate $(20 \mathrm{~mL})$ and extracted with ethyl acetate $(3 \times 20 \mathrm{~mL})$. The combined organic extracts were dried over anhydrous sodium sulphate and evaporated under reduced pressure to give a residue which was purified by silica gel chromatography $(50 \%$, EtOAc/hexanes) to afford $10(520 \mathrm{mg}, 88 \%)$ as a colourless oil. $R_{f}=0.2$ ( $2: 3$ ethyl acetate/hexanes); $[\alpha]^{25}=+79.5$ (c 0.83 , $\mathrm{CHCl}_{3}$ ); IR (neat) 3405, 3098, 2940, 1643, $1068 \mathrm{~cm}^{-1}$; ${ }^{1} \mathrm{H}$ NMR $\left(400 \mathrm{MHz}, \mathrm{CDCl}_{3}\right): \delta 5.71(d d, 1 \mathrm{H}$, $J=17.6,10 \cdot 4 \mathrm{~Hz},=\mathrm{C} \underline{\mathrm{H}}), 5.52-5.44\left(\mathrm{~m}, 2 \mathrm{H}, \mathrm{C}_{2}=\right)$, $4.89(d d, 1 \mathrm{H}, J=9.6,4.0 \mathrm{~Hz}, \mathrm{C} \underline{\mathrm{H}}), 4.48(d, 1 \mathrm{H}$, $J=6.0 \mathrm{~Hz}, \mathrm{C} \underline{\mathrm{H}}), 4.08-4.04(\mathrm{~m}, 1 \mathrm{H}, \mathrm{C} \underline{\mathrm{H}}), 3.92-3.84$ $\left(m, 2 \mathrm{H}, \underline{\mathrm{CH}}_{2}\right), 3.70(d d, 1 \mathrm{H}, J=11 \cdot 2,6.0 \mathrm{~Hz}, \underline{\mathrm{CH}})$, $3.13\left(s, 3 \mathrm{H}, \mathrm{OCH}_{3}\right), 1.46\left(s, 3 \mathrm{H}, \mathrm{C}_{3}\right), 1.32(s, 3 \mathrm{H}$, $\left.\mathrm{C}_{3}\right) ;{ }^{13} \mathrm{C}$ NMR $\left(100 \mathrm{MHz}, \mathrm{CDCl}_{3}\right): \delta 132 \cdot 3,119.9$, $113 \cdot 0,108 \cdot 0,86 \cdot 4,80 \cdot 8,76 \cdot 9,70 \cdot 2,64 \cdot 6,48 \cdot 9,26 \cdot 1$, 24.9; LRMS (ES) $[\mathrm{M}+\mathrm{Na}]^{+} 283 \cdot 1418$; HRMS (ES) 
calcd. for $\mathrm{C}_{12} \mathrm{H}_{20} \mathrm{O}_{6} \mathrm{Na} \mathrm{m} / z$ 283.1158, found $\mathrm{m} / \mathrm{z}$ $283 \cdot 1156$.

2.1b 6-Methoxy-2,2-dimethyl-6-vinyl-tetrahydro-furo [3,4-d]-di-oxole-4-carbaldehyde (11): A suspension of silica supported $\mathrm{NaIO}_{4}(4 \mathrm{~g})$ in $\mathrm{CH}_{2} \mathrm{Cl}_{2}(5 \mathrm{~mL})$ at room temperature was treated with the solution of diol $10(0.5 \mathrm{~g}, 1.9 \mathrm{mmol})$ in $\mathrm{CH}_{2} \mathrm{Cl}_{2}(5 \mathrm{~mL})$ and stirred for $2 \mathrm{~h}$. After the solids were removed by filtration, filtrate was concentrated and the crude compound $\mathbf{1 1}$ was preceded further without purification. $R_{f}=0.6$ ( $1: 1$ ethyl acetate/hexanes).

2.1c 1-(6-Methoxy-2,2-dimethyl-6-vinyl-tetrahydrofuro [3,4-d][1,3]dioxol-4-yl)-but-3-yn-1-ol (12): A suspension of activated zinc and aldehyde 11 (440 mg, $1.71 \mathrm{mmol})$ in THF $(8 \mathrm{~mL})$ at room temperature was treated with a solution of propargyl bromide in THF $(2 \mathrm{~mL})$ and stirred for $3 \mathrm{~h}$. After filtering the reaction mixture through a pad of Celite, the solvent was evaporated; the residue was dissolved in $\mathrm{CH}_{2} \mathrm{Cl}_{2}$ and then quenched with $10 \%$ aqueous $\mathrm{NH}_{4} \mathrm{Cl}$ solution. The aqueous layer was extracted with $\mathrm{CH}_{2} \mathrm{Cl}_{2}$. The organic layer was washed (10\% aqueous $\mathrm{NH}_{4} \mathrm{Cl}$ solution), dried $\left(\mathrm{Na}_{2} \mathrm{SO}_{4}\right)$, concentrated and purified by a flash column chromatography (11\% EtOAc in hexanes) to afford $\mathbf{1 2}$ $(0 \cdot 29 \mathrm{~g}, 67 \%$ over two steps $)$ as a white solid. $R_{f}=0.5$ ( $1: 1$ ethyl acetate/hexanes); m.p. $=70-71^{\circ} \mathrm{C} ;[\alpha]^{25}=$ 74.07 (c 1.08, $\mathrm{CHCl}_{3}$ ); IR (KBr) 3354, 3298, 3021, 2994, 2952, 1375, 1375, $1218 \mathrm{~cm}^{-1}$; ${ }^{1} \mathrm{H}$ NMR $\left(\mathrm{CDCl}_{3}, 300 \mathrm{MHz}\right) \delta 5.71 \quad(d d, \quad 1 \mathrm{H}, \quad J=17 \cdot 4$, $10.8 \mathrm{~Hz},=\mathrm{C} \underline{\mathrm{H}}), 5.53-5.42\left(\mathrm{~m}, 2 \mathrm{H},=\mathrm{C}_{2}\right), 4.91(\mathrm{dd}$, $1 \mathrm{H}, J=5.7,3.9 \mathrm{~Hz}, \mathrm{C} \underline{\mathrm{H}}), 4.48(d, 1 \mathrm{H}, J=5.7 \mathrm{~Hz}$, $\mathrm{C} \underline{\mathrm{H}}), 4 \cdot 14-4.08(m, 1 \mathrm{H}, \mathrm{C} \underline{\mathrm{H}}), 3.88(d d, 1 \mathrm{H}, J=8 \cdot 4$, $3.6 \mathrm{~Hz}, \mathrm{C} \underline{\mathrm{H}}), 3.15\left(\mathrm{~s}, 3 \mathrm{H}, \mathrm{C}_{3}\right), 2.75-2.54(\mathrm{~m}, 2 \mathrm{H}$, $\left.\mathrm{C}_{2}\right), 2.08(t, 1 \mathrm{H}, J=2.7, \equiv \mathrm{C} \underline{\mathrm{H}}), 1.45(s, 3 \mathrm{H}$, $\left.\mathrm{C}_{3}\right), 1.32\left(s, 3 \mathrm{H}, \quad \underline{\mathrm{CH}}_{3}\right) ;{ }^{13} \mathrm{C}$ NMR $\left(\mathrm{CDCl}_{3}\right.$, $75 \mathrm{MHz}) 132 \cdot 2,119.8,113 \cdot 0,107 \cdot 8,86 \cdot 5,80 \cdot 5$, $80 \cdot 4,79 \cdot 6,70 \cdot 8,67 \cdot 8,48 \cdot 9,26 \cdot 1,24 \cdot 8,24 \cdot 4$; LRMS (ES) $[\mathrm{M}+\mathrm{Na}]^{+}$291.1214; HRMS (ES) calcd. for $\mathrm{C}_{14} \mathrm{H}_{20} \mathrm{O}_{5} \mathrm{Na} \mathrm{m} / \mathrm{z} 291 \cdot 1208$, found $\mathrm{m} / \mathrm{z} 291 \cdot 1208$; Anal. calcd. for $\mathrm{C}_{14} \mathrm{H}_{20} \mathrm{O}_{5}$ : C, 62.67; H, 7.51- Found: C, 62.247; H, 7.691.

2.1d (tert-Butyl-[1-(6-methoxy-2,2-dimethyl-6-vinyltetrahydro-furo [3,4-d]dioxol-4-yl]-but-3-ynyloxy]-dimethyl-silane (13): A solution of alcohol $12(0.23 \mathrm{~g}$, $0.86 \mathrm{mmol}$ ) in DMF at room temperature was treated with Immidazole $(0.175 \mathrm{~g}, 2.58 \mathrm{mmol})$, TBSCl $(0.155 \mathrm{~g}, 1.03 \mathrm{mmol})$, catalytic amount of TBAI and then warmed to $50^{\circ} \mathrm{C}$. After stirred for
$24 \mathrm{~h}$ at $50^{\circ} \mathrm{C}$, the reaction mixture was quenched with water and extracted with ethyl acetate. The organic layer was separated, dried $\left(\mathrm{Na}_{2} \mathrm{SO}_{4}\right)$, concentrated and purified by a flash column chromatography ( $4 \%$ EtOAc in hexanes) to afford $13(0.225 \mathrm{~g}, 83 \%)$. $R_{f}=0.7$ ( $1: 9$ ethyl acetate/hexanes); $[\alpha]^{25}=38 \cdot 1$ (c 1.05, $\mathrm{CHCl}_{3}$ ); IR (neat) 3314, 2934, 2857, 1650, $1472 \mathrm{~cm}^{-1}$; ${ }^{1} \mathrm{H}$ NMR $\left(\mathrm{CDCl}_{3}, 300 \mathrm{MHz}\right) 5.68(d d$, $1 \mathrm{H}, J=7.7,10.8 \mathrm{~Hz},=\mathrm{CH}), 5.49-5.39(m, 2 \mathrm{H}$, $\left.=\mathrm{C}_{2}\right), 4.76(d d, 1 \mathrm{H}, J=5.7,3.6 \mathrm{~Hz}, \mathrm{C} \underline{\mathrm{H}}), 4.44(d$, $1 \mathrm{H}, J=5.7 \mathrm{~Hz}, \underline{\mathrm{CH}}), 4.16-4.10(m, 1 \mathrm{H}, \underline{\mathrm{CH}}), 3.92$ $(d d, 1 \mathrm{H}, J=9.3,3.9 \mathrm{~Hz}, \mathrm{C} \underline{\mathrm{H}}), 3 \cdot 15\left(s, 3 \mathrm{H}, \underline{\mathrm{C}}_{3}\right)$, $2.57\left(d d, 2 \mathrm{H}, J=3.6,2.4 \mathrm{~Hz}, \mathrm{C}_{2}\right), 1.97(t, 1 \mathrm{H}$, $J=3.0, \equiv \mathrm{C} \underline{\mathrm{H}}), 1.41 \quad\left(s, 3 \mathrm{H}, \mathrm{C}_{3}\right), 1.28(s, 3 \mathrm{H}$, $\left.\mathrm{C}_{\mathrm{H}_{3}}\right), 0.9\left(s, 9 \mathrm{H}, 3 \times \mathrm{C}_{3}\right), 0 \cdot 13\left(s, 3 \mathrm{H}, \mathrm{CH}_{3}\right), 0 \cdot 10$ $\left(s, 3 \mathrm{H}, \mathrm{CH}_{3}\right) ;{ }^{13} \mathrm{C} \mathrm{NMR}\left(\mathrm{CDCl}_{3}, 75 \mathrm{MHz}\right) 132.7$, $119 \cdot 3,112 \cdot 3,107 \cdot 7,86 \cdot 7,81 \cdot 3,80 \cdot 0,79 \cdot 6,70 \cdot 05$, $67 \cdot 5,48 \cdot 9,26 \cdot 1,25 \cdot 9,25 \cdot 1,24 \cdot 9,18 \cdot 2,-4 \cdot 6,-4 \cdot 8$; HRMS (ES) calcd. for $\mathrm{C}_{20} \mathrm{H}_{34} \mathrm{O}_{5} \mathrm{NaSi} m / z$ 405.2073, found $m / z$ 405.2091; Anal. calcd. for $\mathrm{C}_{20} \mathrm{H}_{34} \mathrm{O}_{5} \mathrm{Si}$ : C, 62.79; H, 8.96. Found: C, 62.083; H, 9.282.

2.1e (tert-Butyl-[1-(6-methoxy-2,2-dimethyl-6-vinyltetrahydro-furo [3,4-d]dioxol-4-yl]-3-methylene-pent4-enyloxy]dime-thyl-silane (14): Enyne 13 (0.078 g, $0.2 \mathrm{mmol})$ was dissolved in $\mathrm{CH}_{2} \mathrm{Cl}_{2}(62 \mathrm{~mL})$ and ethylene gas was purged for $20 \mathrm{~min}$. A solution of catalyst $17(5 \mathrm{~mol} \%)$ in $4 \mathrm{~mL}$ of $\mathrm{CH}_{2} \mathrm{Cl}_{2}$ was added slowly at room temperature and stirred for $3 \mathrm{~h}$. The reaction mixture was filtered, concentrated and purified by a flash column chromatography $(0.5 \%$ EtOAc in hexanes) to afford $14(0.051 \mathrm{~g}, 64 \%) . R_{f}=$ 0.8 ( $1: 24$ ethyl acetate/hexanes); IR (neat) 3020, $1650,1216 \mathrm{~cm}^{-1}$; ${ }^{1} \mathrm{H}$ NMR $\left(\mathrm{CDCl}_{3}, 300 \mathrm{MHz}\right) \delta$ $6.39(d d, 1 \mathrm{H}, J=18,11.2 \mathrm{~Hz},=\mathrm{C} \underline{\mathrm{H}}), 5.69(d d, 1 \mathrm{H}$, $J=10.8, \quad 17.6 \mathrm{~Hz}, \quad=\mathrm{C} \underline{\mathrm{H}}), \quad 5.50-5.30 \quad(\mathrm{~m}, \quad 3 \mathrm{H}$, $3 \times=\mathrm{C} \underline{\mathrm{H}}), 5.15-5.06(\mathrm{~m}, 3 \mathrm{H}, 3 \times \mathrm{C} \underline{\mathrm{H}}), 4.75(\mathrm{dd}$, $1 \mathrm{H}, J=5.6,3.6 \mathrm{~Hz},=\mathrm{C} \underline{\mathrm{H}}), 4.44(d, 1 \mathrm{H}, J=5.6 \mathrm{~Hz}$, $\mathrm{C} \underline{\mathrm{H}}$ of $\left.\mathrm{CH}_{2}\right), 4 \cdot 3(d d d, 1 \mathrm{H}, J=16 \cdot 4,8 \cdot 4,2 \cdot 8 \mathrm{~Hz}, \mathrm{C} \underline{\mathrm{H}}$ of $\left.\mathrm{CH}_{2}\right), 3 \cdot 71(d d, 1 \mathrm{H}, J=7 \cdot 6,3 \cdot 2 \mathrm{~Hz}, \mathrm{CH}), 3 \cdot 11(s$, $\left.3 \mathrm{H}, \underline{\mathrm{C}}_{3}\right), 2.9(d, 1 \mathrm{H}, J=14.4 \mathrm{~Hz}, \mathrm{C} \underline{\mathrm{H}}), 4.44(d d$, $1 \mathrm{H}, J=14.4,8.4 \mathrm{~Hz}, \mathrm{C} \underline{\mathrm{H}}), 1.40\left(s, 3 \mathrm{H}, \mathrm{C}_{3}\right), 1.27$ $\left(s, 3 \mathrm{H}, \mathrm{CH}_{3}\right), 0.86\left(s, 9 \mathrm{H}, 3 \times \underline{\mathrm{CH}}_{3}\right), 0.07(s, 6 \mathrm{H}$, $\left.2 \times \mathrm{CH}_{3}\right) ;{ }^{13} \mathrm{C}$ NMR $\left(\mathrm{CDCl}_{3}, 75 \mathrm{MHz}\right) 143 \cdot 0,139 \cdot 1$, $132 \cdot 8,119 \cdot 4,119 \cdot 0,113 \cdot 8,112 \cdot 4,107 \cdot 5,86 \cdot 7,81 \cdot 9$, $80 \cdot 1,68 \cdot 4,48 \cdot 9,37 \cdot 9,26 \cdot 2,24 \cdot 9,18 \cdot 3,1 \cdot 1,-3 \cdot 7$, -4.8; LRMS (ES) $[\mathrm{M}+\mathrm{Na}]^{+}$433.3003; HRMS (ES) calcd. for $\mathrm{C}_{22} \mathrm{H}_{39} \mathrm{O}_{5} \mathrm{Si}[\mathrm{M}+1]^{+} \mathrm{m} / z$ 411.2570, found $m / z 411.2567$.

2.1f (5-Ethynyl-2,2-dimethyl-[1,3]dioxolan-4-yl)-methanol (22): A solution of D (+) ribose acetonide 21 
$(10 \mathrm{~g}, 52.3 \mathrm{mmol})$ in $\mathrm{MeOH}(250 \mathrm{~mL})$ was treated with $\mathrm{NaBH}_{4}(8.1 \mathrm{~g}, 213 \mathrm{mmol})$ in portion-wise at $0^{\circ} \mathrm{C}$ and the stirring was continued for $2 \mathrm{~h}$ before neutralizing with glacial $\mathrm{AcOH}$ at $0^{\circ} \mathrm{C}$. After adjusting the $\mathrm{pH}$ to 7 , water $(150 \mathrm{~mL})$ was added and treated with finely powdered $\mathrm{NaIO}_{4}(20 \mathrm{~g})$ at room temperature and the stirring was continued for further $3 \mathrm{~h}$. After filtration, the solvent was evaporated and then the residue was extracted with ethyl acetate. The organic layer was washed with brine, dried $\left(\mathrm{Na}_{2} \mathrm{SO}_{4}\right)$, concentrated and purified by silica gel chromatography (25\% EtOAc in hexanes) to afford the lactol $22(7.2 \mathrm{~g})$ as a colourless oil in $86 \%$ yield. $R_{f}=0.53$ (1: 1 ethyl acetate/hexanes).

A mixture of lactol $22(5 \mathrm{~g}, 31.2 \mathrm{mmol}), \mathrm{K}_{2} \mathrm{CO}_{3}$ (13 g, $93.6 \mathrm{mmol}$ ) was heated to reflux. When the reflux initiated, dimethyl-1-diazo-2-oxopropylphosphonate 23 (20 g, $93.6 \mathrm{mmol})$ was added drop-wise over a period of $6 \mathrm{~h}$ using a syringe pump. The reaction mixture was cooled to room temperature and the stirring was continued for further $12 \mathrm{~h}$. Filtered, solvent was evaporated, treated with water and then extracted with ethyl acetate. The organic layer was washed with brine, dried $\left(\mathrm{Na}_{2} \mathrm{SO}_{4}\right)$, concentrated and purified by silica gel chromatography $(22 \%$ EtOAc in hexanes) to afford the alcohol $24(3.7 \mathrm{~g})$ as a colourless oil in $76 \%$ yield. $R_{f}=0.5(1: 1$ ethyl acetate/ hexanes); $[\alpha]_{\mathrm{D}}^{25}=-35.29\left(c=1 \cdot 36, \mathrm{CHCl}_{3}\right)$; IR (neat) $3306, \quad 2937, \quad 1457 \mathrm{~cm}^{-1} ;{ }^{1} \mathrm{H} \quad \mathrm{NMR} \quad(300 \mathrm{MHz}$, $\left.\mathrm{CDCl}_{3}\right): \delta 4.6(d d, 1 \mathrm{H}, J=7.5,2 \cdot 1 \mathrm{~Hz}, \mathrm{CH}), 4 \cdot 19$ $(d t, 1 \mathrm{H}, J=7.5,3.3 \mathrm{~Hz}, \mathrm{C} \underline{\mathrm{H}}), 3.91(d d, 1 \mathrm{H}, J=3 \cdot 0$, $12.6 \mathrm{~Hz}, \mathrm{CH}$ of $\left.\mathrm{CH}_{2}\right), 3.69(d d, 1 \mathrm{H}, J=3.6$, $12.6 \mathrm{~Hz}, \underline{\mathrm{CH}}$ of $\left.\mathrm{CH}_{2}\right), 2.55(d, 1 \mathrm{H}, J=2.1 \mathrm{~Hz}, \equiv \mathrm{C} \underline{\mathrm{H}})$, $1.51\left(s, 3 \mathrm{H}, \mathrm{C}_{3}\right), 1.44\left(s, 3 \mathrm{H}, \mathrm{C}_{3}\right) ;{ }^{13} \mathrm{C} \mathrm{NMR}$ $\left(75 \mathrm{MHz}, \mathrm{CDCl}_{3}\right): \delta 110 \cdot 7,82 \cdot 0,80 \cdot 7,74 \cdot 9,66 \cdot 2,60 \cdot 7$, 26.7, 26.1; LRMS (ES) $[\mathrm{M}+\mathrm{Na}]^{+} 179 \cdot 1115$; HRMS (ES) calcd. for $\mathrm{C}_{8} \mathrm{H}_{12} \mathrm{O}_{3} \mathrm{Na} \mathrm{m} / z \quad 179.0684$, found $\mathrm{m} / \mathrm{z}$ 179.0677.

\section{1g Tert-Butyl-(5-ethynyl-2,2-dimethyl-[1,3]dioxo-} lan-4-ylmethoxy)-dimethyl-silane (25): A solution of alcohol 24 ( $2.5 \mathrm{~g}, 19.23 \mathrm{mmol})$, imidazole (1.63 g, $24.03 \mathrm{mmol}$ ) and catalytic amount of DMAP in $\mathrm{CH}_{2} \mathrm{Cl}_{2}(50 \mathrm{~mL})$ was treated with TBSCl $(2.9 \mathrm{~g}$, $19.23 \mathrm{mmol})$ at room temperature. After being stirred at room temperature for $2 \mathrm{~h}$, the reaction mixture was treated with water and extracted with $\mathrm{CH}_{2} \mathrm{Cl}_{2}$. The organic layer was washed with brine, dried $\left(\mathrm{Na}_{2} \mathrm{SO}_{4}\right)$, concentrated and purified by silica gel chromatography (2\% EtOAc in hexanes) to afford the silyl ether $25(3.8 \mathrm{~g})$ as a colourless oil in $88 \%$ yield.
$R_{f}=0.81 \quad(1: 9$ ethyl acetate/hexanes $) ;[\alpha]^{25}=$ $-14.52\left(c=1 \cdot 17, \mathrm{CHCl}_{3}\right)$; IR (neat) 3019, 2930, 2858, $2030 \mathrm{~cm}^{-1} ;{ }^{1} \mathrm{H}$ NMR (300 MHz, $\left.\mathrm{CDCl}_{3}\right): \delta 4 \cdot 6$ $(d d, 1 \mathrm{H}, J=7 \cdot 2,2 \cdot 1 \mathrm{~Hz}, \mathrm{C} \underline{\mathrm{H}}), 4 \cdot 12(d t, 1 \mathrm{H}, J=7 \cdot 2$, $3.9 \mathrm{~Hz}, \mathrm{C} \underline{\mathrm{H}}), 3.9\left(d, 2 \mathrm{H}, J=3.9 \mathrm{~Hz}, \mathrm{CH}_{2}\right), 2.52(d$, $1 \mathrm{H}, J=2.1 \mathrm{~Hz}, \equiv \mathrm{C} \underline{\mathrm{H}}), 1.49\left(\mathrm{~s}, 3 \mathrm{H}, \mathrm{CH}_{3}\right), 1.41(\mathrm{~s}$, $\left.3 \mathrm{H}, \mathrm{CH}_{3}\right), 0.90\left(s, 9 \mathrm{H}, 3 \times \mathrm{CH}_{3}\right), 0.08(s, 6 \mathrm{H}$, $\left.2 \times \mathrm{CH}_{3}\right) ;{ }^{13} \mathrm{C}$ NMR $\left(100 \mathrm{MHz}, \mathrm{CDCl}_{3}\right): \delta 110 \cdot 7$, $82 \cdot 4,81 \cdot 4,74 \cdot 5,67 \cdot 1,62 \cdot 1,27 \cdot 0,26 \cdot 4,26 \cdot 0,18 \cdot 5$, $-5 \cdot 2,-5 \cdot 3$; LRMS (ES) $[\mathrm{M}+\mathrm{Na}]^{+} 293 \cdot 1760$; HRMS (ES) calcd. for $\mathrm{C}_{14} \mathrm{H}_{26} \mathrm{O}_{3} \mathrm{NaSi} m / z 293 \cdot 1549$, found $\mathrm{m} / z$ 293.1547.

\section{$2.1 \mathrm{~h}$ Tert-Butyl-(5-ethynyl-2,2-dimethyl-[1,3]dioxo-} lan-4-yl-methoxy)-dimethyl-silane (26): A solution of alkyne $25(3.74 \mathrm{~g}, 13.9 \mathrm{mmol})$ in THF $(65 \mathrm{~mL})$ was treated with ${ }^{n} \mathrm{BuLi}(11.3 \mathrm{~mL}, 18 \mathrm{mmol}, 1.6 \mathrm{M}$ in hexane) at $-78^{\circ} \mathrm{C}$ and after being stirred for $30 \mathrm{~min}$. the reaction mixture was treated with HMPA $(4.83 \mathrm{~mL}$, $27.8 \mathrm{mmol})$ at $-78^{\circ} \mathrm{C}$ followed by MeI $(1.7 \mathrm{~mL}$, $27.8 \mathrm{mmol})$. The stirring was continued at the same temperature for $20 \mathrm{~min}$. and then slowly warmed to room temperature. After being stirred at room temperature for $12 \mathrm{~h}$, the reaction mixture was quenched with saturated $\mathrm{NH}_{4} \mathrm{Cl}$ solution and extracted with ethyl acetate. The organic layer was washed with brine, dried $\left(\mathrm{Na}_{2} \mathrm{SO}_{4}\right)$, concentrated and purified by silica gel chromatography ( $2 \%$ EtOAc in hexanes) to afford the silyl ether $26(3.76 \mathrm{~g})$ in $96 \%$ yield as a colourless oil. $R_{f}=0.27$ (hexanes); $[\alpha]^{25}=-28.85$ (c $1.04, \mathrm{CHCl}_{3}$ ); IR (neat) 2931, 2246, 1802, $1257 \mathrm{~cm}^{-1} ;{ }^{1} \mathrm{H}$ NMR $\left(300 \mathrm{MHz}, \mathrm{CDCl}_{3}\right): \delta 4.55(d q$, $1 \mathrm{H}, J=7.8,2.1 \mathrm{~Hz}, \mathrm{C} \underline{\mathrm{H}}), 4.01(d t, 1 \mathrm{H}, J=7.2$, $3.9 \mathrm{~Hz}, \mathrm{C} \underline{\mathrm{H}}), 3.77\left(d d, 2 \mathrm{H}, J=3.9,2.1 \mathrm{~Hz}, \mathrm{C}_{2}\right)$, $1.86\left(d, 3 \mathrm{H}, J=2.1 \mathrm{~Hz}, \mathrm{C}_{3}\right), 1.48\left(s, 3 \mathrm{H}, \mathrm{C}_{3}\right)$, $1.39\left(s, 3 \mathrm{H}, \mathrm{C}_{3}\right), 0.91\left(s, 9 \mathrm{H}, 3 \times \mathrm{CH}_{3}\right), 0.08(s$, $\left.6 \mathrm{H}, 2 \times \mathrm{CH}_{3}\right) ;{ }^{13} \mathrm{C}$ NMR $\left(75 \mathrm{MHz}, \mathrm{CDCl}_{3}\right): \delta 109 \cdot 9$, $83 \cdot 0,82 \cdot 2,76 \cdot 0,67 \cdot 5,62 \cdot 0,26 \cdot 9,26 \cdot 6,25 \cdot 9,18 \cdot 4$, 3.8; LRMS (ES) $[\mathrm{M}+\mathrm{Na}]^{+}$307.2350; HRMS (ES) calcd. for $\mathrm{C}_{15} \mathrm{H}_{28} \mathrm{O}_{3} \mathrm{NaSi} \mathrm{m} / \mathrm{z} 307 \cdot 1705$, found $\mathrm{m} / \mathrm{z}$ $307 \cdot 1692$.

2.1i (2,2-Dimethyl-5-prop-1-ynyl-[1,3]dioxolan-4-yl)methanol (20): A solution of silyl ether $26(3.7 \mathrm{~g}$, $13.02 \mathrm{mmol})$ in THF $(75 \mathrm{~mL})$ was treated with a solution of TBAF $(5.11 \mathrm{~g}, 19.5 \mathrm{mmol})$ in THF $(25 \mathrm{~mL})$ at $0^{\circ} \mathrm{C}$. After being stirred at $0^{\circ} \mathrm{C}$ for $15 \mathrm{~min}$., the reaction mixture was allowed to stir at room temperature for $2 \mathrm{~h}$. The reaction mixture was treated with water and extracted with ethyl acetate. The organic layer was washed with brine, dried $\left(\mathrm{Na}_{2} \mathrm{SO}_{4}\right)$, con- 
centrated and purified by silica gel chromatography $(20 \%$ EtOAc in hexanes) to afford the alcohol 20 $(2.05 \mathrm{~g})$ in $93 \%$ yield as a colourless oil. $R_{f}=0.15$ ( $2: 8$ ethyl acetate/hexanes); $[\alpha]^{25}{ }_{\mathrm{D}}=-19.31$ (c 1.45 , $\mathrm{CHCl}_{3}$ ); IR (neat) $3437,2991,2247,1218 \mathrm{~cm}^{-1} ;{ }^{1} \mathrm{H}$ NMR $\left(300 \mathrm{MHz}, \mathrm{CDCl}_{3}\right): \delta 4.55(d q, 1 \mathrm{H}, J=7 \cdot 8$, $2 \cdot 1 \mathrm{~Hz}, \mathrm{C} \underline{\mathrm{H}}), 4.08(d t, 1 \mathrm{H}, J=7 \cdot 8,3.0 \mathrm{~Hz}, \underline{\mathrm{CH}}), 3.9$ $\left(d d, 1 \mathrm{H}, J=3.0,12 \mathrm{~Hz}, \mathrm{C} \underline{\mathrm{H}}\right.$ of $\left.\mathrm{CH}_{2}\right), 3.67(d d, 1 \mathrm{H}$, $J=3 \cdot 0,12 \mathrm{~Hz}, \mathrm{C} \underline{\mathrm{H}}$ of $\left.\mathrm{CH}_{2}\right), 2 \cdot 09(b r s, 1 \mathrm{H}, \mathrm{OH})$, $1.87\left(d, 3 \mathrm{H}, J=2.1 \mathrm{~Hz}, \underline{\mathrm{CH}}_{3}\right), 1.5\left(s, 3 \mathrm{H}, \underline{\mathrm{C}}_{3}\right), 1.45$ $\left(s, 3 \mathrm{H}, \mathrm{CH}_{3}\right) ;{ }^{13} \mathrm{C}$ NMR $\left(75 \mathrm{MHz}, \mathrm{CDCl}_{3}\right): \delta 110 \cdot 0$, $83 \cdot 4,81 \cdot 9,75 \cdot 4,66 \cdot 8,61 \cdot 0,26 \cdot 8,26 \cdot 4,3 \cdot 7$; HRMS (ES) calcd. for $\mathrm{C}_{9} \mathrm{H}_{14} \mathrm{O}_{3} \mathrm{Na} \mathrm{m} / z$ 193.0841, found $\mathrm{m} / \mathrm{z}$ $193 \cdot 0836$.

$2.1 \mathrm{j}$ 4-Iodomethyl-2,2-dimethyl-5-prop-1-ynyl-[1,3] dioxolane (27): To a solution of alcohol $20(0.540 \mathrm{~g}$, $3.17 \mathrm{mmol})$ in pyridine $(15 \mathrm{~mL})$ was added $p-\mathrm{TsCl}$ $(1.2 \mathrm{~g}, 6.4 \mathrm{mmol})$ at $0^{\circ} \mathrm{C}$. The stirring was continued at the same temperature for $20 \mathrm{~min}$ and then at room temperature for $12 \mathrm{~h}$. The reaction mixture was treated with water and extracted with ethyl acetate. The organic layer was washed with brine, dried $\left(\mathrm{Na}_{2} \mathrm{SO}_{4}\right)$, concentrated and purified by silica gel chromatography (15\% EtOAc in hexanes) to afford the tosyl ether $(0.89 \mathrm{~g})$ as a colourless oil in $87 \%$ yield.

$R_{f}=0.34$ (2:8 ethyl acetate/hexanes); $[\alpha]^{25}{ }_{\mathrm{D}}=-$ 52.884 (c 1.04, $\mathrm{CHCl}_{3}$ ); IR (neat) 3024, 1455, 1373 $\mathrm{cm}^{-1} ;{ }^{1} \mathrm{H}$ NMR $\left(300 \mathrm{MHz}, \mathrm{CDCl}_{3}\right): \delta 7.81(d, 2 \mathrm{H}$, $J=7.8,2 \times \mathrm{CH}=$ of $\mathrm{Ph}), 7.36(d, 2 \mathrm{H}, J=7.8$, $2 \times \mathrm{C} \underline{\mathrm{H}}=$ of $\mathrm{Ph}), 4.44(d q, 1 \mathrm{H}, J=9 \cdot 0,2 \cdot 1 \mathrm{~Hz}, \mathrm{C} \underline{\mathrm{H}})$, 4.25-4.06 $\left(\mathrm{m}, 3 \mathrm{H}, 1 \times \mathrm{CH}\right.$ and $\left.\mathrm{C}_{2}\right), 2.5(s, 3 \mathrm{H}$, $\left.\mathrm{C}_{3}\right), 1.84\left(d, 3 \mathrm{H}, J=2.1 \mathrm{~Hz}, \underline{\mathrm{CH}}_{3}\right), 1.44(s, 3 \mathrm{H}$, $\left.\mathrm{C}_{3}\right), 1.32\left(s, 3 \mathrm{H}, \mathrm{C}_{3}\right) ;{ }^{13} \mathrm{C}$ NMR $\left(75 \mathrm{MHz}, \mathrm{CDCl}_{3}\right)$ : $\delta 145 \cdot 1,132 \cdot 8,129 \cdot 9,128 \cdot 1,110 \cdot 9,84 \cdot 0,79 \cdot 1,74 \cdot 8$, $67 \cdot 8,67 \cdot 4,26 \cdot 7,26 \cdot 5,21 \cdot 7,3 \cdot 7 \cdot$ HRMS (ES) calcd. for $\mathrm{C}_{16} \mathrm{H}_{20} \mathrm{O}_{5} \mathrm{SNa} m / z$ 347.0929, found 347.0941.

A mixture of tosyl ether $(0.84 \mathrm{~g}, 2.6 \mathrm{mmol})$ and $\mathrm{NaI}(1.36 \mathrm{~g}, 9.06 \mathrm{mmol})$ in ethyl methyl ketone $(87 \mathrm{~mL})$ was heated to reflux for $12 \mathrm{~h}$. The solvent was evaporated, treated with water and extracted with ethyl acetate. The organic layer was washed with thio solution, water, brine, dried $\left(\mathrm{Na}_{2} \mathrm{SO}_{4}\right)$, concentrated and purified by silica gel chromatography (4\% EtOAc in hexanes) to afford the iodo compound 27 $(0 \cdot 6 \mathrm{~g})$ as a colourless oil in $83 \%$ yield. $R_{f}=0.4$ ( $2: 8$ ethyl acetate/hexanes); $[\alpha]^{25}=-48 \cdot 17$ (c 1.64, $\mathrm{CHCl}_{3}$ ); IR (neat) 2990, 2922, 2250, 1455, $1381 \mathrm{~cm}^{-1}$; ${ }^{1} \mathrm{H}$ NMR $\left(300 \mathrm{MHz}, \mathrm{CDCl}_{3}\right): \delta 4.40(d q, 1 \mathrm{H}, J=4.5$, $1.8 \mathrm{~Hz}, \mathrm{C} \underline{\mathrm{H}}), 3.92(d t, 1 \mathrm{H}, J=10.5,4.5 \mathrm{~Hz}, \mathrm{C} \underline{\mathrm{H}})$, $3.37\left(d d, 1 \mathrm{H}, J=10 \cdot 5,5 \cdot 1 \mathrm{~Hz}, \mathrm{CH}\right.$ of $\left.\mathrm{CH}_{2}\right), 3 \cdot 28$ $\left(d d, 1 \mathrm{H}, J=10.5,5 \cdot 1 \mathrm{~Hz}, \mathrm{C} \underline{\mathrm{H}}\right.$ of $\left.\mathrm{CH}_{2}\right), 1.88(d, 3 \mathrm{H}$, $\left.J=1.8 \mathrm{~Hz}, \mathrm{CH}_{3}\right), 1.49\left(s, 3 \mathrm{H}, \mathrm{CH}_{3}\right), 1.46(s, 3 \mathrm{H}$, $\left.\mathrm{CH}_{3}\right) ;{ }^{13} \mathrm{C}$ NMR $\left(75 \mathrm{MHz}, \mathrm{CDCl}_{3}\right): \delta 110 \cdot 7,84 \cdot 0$, $80 \cdot 4,75 \cdot 4,71 \cdot 4,27 \cdot 3,26 \cdot 8,4 \cdot 9,3 \cdot 8$.

2.1k 3-(4S,5R)-2,2-Dimethyl-5-(prop-1-ynyl)-1,3-dioxolan-4-yl)propanenitrile (29): To a solution of $\mathrm{CH}_{3} \mathrm{CN}(0.133 \mathrm{~mL}, 2.55 \mathrm{mmol})$ in THF $(3 \mathrm{~mL})$ at $-78^{\circ} \mathrm{C}$ was added ${ }^{n} \mathrm{BuLi}(1.01 \mathrm{~mL}, 1.1 \mathrm{M}$ in hexane) drop wise. After stirring at the same temperature for $1 \mathrm{~h}$, a solution of $27(0.285 \mathrm{~g}, 1.02 \mathrm{mmol})$ in THF $(1.3 \mathrm{~mL})$ was added and continued the stirring for another $2 \mathrm{~h}$. The reaction mixture was slowly warmed to room temperature and treated with saturated $\mathrm{NH}_{4} \mathrm{Cl}$ solution. The reaction mixture was extracted with ether. The organic layer was washed with water, brine, dried $\left(\mathrm{Na}_{2} \mathrm{SO}_{4}\right)$, concentrated and purified by silica gel chromatography (4\% EtOAc in hexanes) to afford the nitrile $29(0.042 \mathrm{~g})$ as colourless oil in $21 \%$ yield along with starting material $27(0 \cdot 104 \mathrm{~g})$. $R_{f}=0.4 \quad(1: 19$ ethyl acetate/hexanes); IR (neat) 2989, 2934, 2853, 2248, 1442, 1373, 1239, $1160 \mathrm{~cm}^{-1}$. ${ }^{1} \mathrm{H}$ NMR $\left(300 \mathrm{MHz}, \mathrm{CDCl}_{3}\right): \delta 4 \cdot 22(d q, 1 \mathrm{H}, J=8 \cdot 0$, $2.0 \mathrm{~Hz}, \mathrm{CH}), 4.00(d t, 1 \mathrm{H}, J=8.0,3.6 \mathrm{~Hz}, \mathrm{CH}), 2.57-$ $2.49\left(m, 2 \mathrm{H}, \mathrm{CH}_{2}\right), 2.11-2.02(m, 1 \mathrm{H}, \mathrm{CH}), 1.92-$ $1.83(m, 1 \mathrm{H}, \mathbf{C H}), 1.88\left(d, 3 \mathrm{H}, J=2.0 \mathrm{~Hz}, \mathrm{CH}_{3}\right)$, $1.46\left(s, 3 \mathrm{H}, \mathrm{CH}_{3}\right), 1.39\left(s, 3 \mathrm{H}, \mathrm{CH}_{3}\right) ;{ }^{13} \mathrm{C} \mathrm{NMR}$ $\left(75 \mathrm{MHz}, \mathrm{CDCl}_{3}\right): \delta 119 \cdot 1,110 \cdot 1,84 \cdot 1,78 \cdot 5,74 \cdot 7$, $70 \cdot 5,28 \cdot 0,27 \cdot 1,26 \cdot 5,14 \cdot 1,3 \cdot 8$; HRMS (ES) calcd. for $\mathrm{C}_{11} \mathrm{H}_{15} \mathrm{O}_{2} \mathrm{NNa} m / z$ 216.0998, found $m / z$ 216.0998.

\subsection{3-(2,2-Dimethyl-5-prop-1-ynyl-[1,3]dioxolan-}

4-yl)-acrylic acid ethyl ester (30): To a solution of oxalyl chloride $(0.62 \mathrm{~mL}, 7.06 \mathrm{mmol})$ in $\mathrm{CH}_{2} \mathrm{Cl}_{2}$ $(20 \mathrm{~mL})$ was added DMSO $(1.0 \mathrm{~mL}, 14.11 \mathrm{mmol})$ at $-78^{\circ} \mathrm{C}$ and after being stirred at the same temperature for $10 \mathrm{~min}$. a solution of alcohol $20(1.0 \mathrm{~g}$, $5.88 \mathrm{mmol})$ in $\mathrm{CH}_{2} \mathrm{Cl}_{2}(9 \mathrm{~mL})$ was added drop wise and continuously stirred for $1 \mathrm{~h}$. Finally triethylamine $(4.3 \mathrm{~mL}, 31.0 \mathrm{mmol})$ was added and the reaction mixture was stirred at $-78^{\circ} \mathrm{C}$ for $20 \mathrm{~min}$. and then gradually warmed to room temperature. The reaction mixture was treated with water and extracted with $\mathrm{CH}_{2} \mathrm{Cl}_{2}$. The organic layer was washed with brine, dried $\left(\mathrm{Na}_{2} \mathrm{SO}_{4}\right)$, concentrated and the crude product was used for further reaction without any purification. $R_{f}=0.31$ (40\% EtOAc in hexanes); IR (neat) $3020,2250,1736,1383,1217 \mathrm{~cm}^{-1}$.

A solution of carboethoxymethylenetriphenyl phosphorane $(2.95 \mathrm{~g}, 8.82 \mathrm{mmol})$ in acetonitrile 
$(28 \mathrm{~mL})$ was treated with a solution of above aldehyde in acetonitrile $(14 \mathrm{~mL})$ at room temperature and the stirring was continued for $2 \mathrm{~h}$. The solvent was evaporated and the resulting liquid was purified by column chromatography (4\% EtOAc in hexanes) to afford the unsaturated ester $30(0.83 \mathrm{~g})$ as a colourless oil in $68 \%$ yield for two steps. $R_{f}=0.78$ (3:7 ethyl acetate/hexanes); $[\alpha]^{25}{ }_{\mathrm{D}}=-112.72 \quad(c) 1 \cdot 1$, $\mathrm{CHCl}_{3}$ ); IR (neat) 2989.5, 1722, 1375, $1051 \mathrm{~cm}^{-1}$; ${ }^{1} \mathrm{H}$ NMR $\left(400 \mathrm{MHz}, \mathrm{CDCl}_{3}\right): \delta 6.91(d d, 1 \mathrm{H}, J=15 \cdot 6$, $5.6 \mathrm{~Hz}, \mathrm{C} \underline{\mathrm{H}}=), 6.19(d d, 1 \mathrm{H}, J=15.6,1.6, \mathrm{C} \underline{\mathrm{H}}=)$, $4.49(d q, 1 \mathrm{H}, J=8.0,1.6 \mathrm{~Hz}, \mathrm{CH}), 4.3(d q, 1 \mathrm{H}$, $J=8.4,2 \mathrm{~Hz}, \mathrm{C} \underline{\mathrm{H}}), 4.22\left(q, 2 \mathrm{H}, J=7.2 \mathrm{~Hz}, \mathrm{C}_{2}\right)$, $1.89\left(d, 3 \mathrm{H}, J=1.9 \mathrm{~Hz}, \mathrm{C}_{3}\right), 1.50\left(s, 3 \mathrm{H}, \underline{C}_{3}\right)$, $1.42\left(s, 3 \mathrm{H}, \mathrm{CH}_{3}\right), 1.31\left(t, 3 \mathrm{H}, J=7.2, \mathrm{CH}_{3}\right) ;{ }^{13} \mathrm{C}$ NMR $\left(75 \mathrm{MHz}, \mathrm{CDCl}_{3}\right): \delta 165.9,142.2,123.4$, $110 \cdot 6,84 \cdot 3,80 \cdot 6,74 \cdot 0,70 \cdot 7,60 \cdot 7,26 \cdot 8,26 \cdot 7,14 \cdot 3$, 3.8; HRMS (ES) calcd. for $\mathrm{C}_{13} \mathrm{H}_{18} \mathrm{O}_{4} \mathrm{Na} \mathrm{m} / z$ $261 \cdot 1103$, found $m / z 261 \cdot 1102$.

$2.1 \mathrm{~m}$ 3-(2,2-Dimethyl-5-prop-1-ynyl-[1,3]dioxolan4-yl)-propionic acid ethyl ester (31): A suspension of $\mathrm{Cu}_{2} \mathrm{Cl}_{2}(0.21 \mathrm{~g}, 2.14 \mathrm{mmol})$ and the ester 30 $(0.68 \mathrm{~g}, 2.85 \mathrm{mmol})$ in THF $(40 \mathrm{~mL})$ and $\mathrm{MeOH}$ $(17 \mathrm{~mL})$ at $-20^{\circ} \mathrm{C}$ was treated with $\mathrm{NaBH}_{4}(0.65 \mathrm{~g}$, $17.12 \mathrm{mmol}$ ) in portion-wise over a period of $10 \mathrm{~min}$. The resultant black suspension was stirred for an additional $30 \mathrm{~min}$ at the same temperature. The black precipitate was filtered off and the filtrate was concentrated. The slurry was treated with saturated $\mathrm{NH}_{4} \mathrm{Cl}$ solution and extracted with ether. The organic layer was washed with water, brine, dried $\left(\mathrm{Na}_{2} \mathrm{SO}_{4}\right)$, concentrated and purified by silica gel chromatography (4\% EtOAc in hexanes) to afford the saturated ester $31(0.55 \mathrm{~g})$ in $80 \%$ yield. $R_{f}=0.55(2: 8$ ethyl acetate/hexanes); $[\alpha]_{\mathrm{D}}^{25}=-14.63\left(\mathrm{c} 1.23, \mathrm{CHCl}_{3}\right)$; IR (neat) 2987, 2935, 2250, 1732, 1455, $1372 \mathrm{~cm}^{-1}$; ${ }^{1} \mathrm{H}$ NMR $\left(400 \mathrm{MHz}, \mathrm{CDCl}_{3}\right): \delta 4.21(d q, 1 \mathrm{H}$, $J=8 \cdot 0,2 \cdot 4 \mathrm{~Hz}, \mathrm{C} \underline{\mathrm{H}}), 4 \cdot 15\left(q, 2 \mathrm{H}, J=7.2 \mathrm{~Hz}, \underline{\mathrm{C}}_{2}\right)$, $4.15(q, 1 \mathrm{H}, J=6.8 \mathrm{~Hz}, \mathrm{C} \underline{\mathrm{H}}), 2.57-2.42(m, 2 \mathrm{H}$, $\left.\mathrm{CH}_{2}\right), 2.04-2.01\left(\mathrm{~m}, 1 \mathrm{H}, \mathrm{CH}\right.$ of $\left.\mathrm{CH}_{2}\right), 1.93-1.87(\mathrm{~m}$, $1 \mathrm{H}, \mathrm{CH}$ of $\left.\mathrm{CH}_{2}\right), 1.86\left(d, 3 \mathrm{H}, J=1.6 \mathrm{~Hz}, \mathrm{CH}_{3}\right)$, $1.44\left(s, 3 \mathrm{H}, \mathrm{C}_{3}\right), 1.38\left(s, 3 \mathrm{H}, \mathrm{CH}_{3}\right), 1.27(t, 3 \mathrm{H}$, $\left.J=7.2 \mathrm{~Hz}, \mathrm{C}_{3}\right) ;{ }^{13} \mathrm{C}$ NMR $\left(75 \mathrm{MHz}, \mathrm{CDCl}_{3}\right) \delta$ $173 \cdot 1,109 \cdot 6,83 \cdot 5,80 \cdot 5,75 \cdot 2,70 \cdot 7,60 \cdot 6,30 \cdot 5,27 \cdot 2$, $26 \cdot 5,14 \cdot 3,3 \cdot 8$; HRMS (ES) calcd. for $\mathrm{C}_{13} \mathrm{H}_{20} \mathrm{O}_{4} \mathrm{Na}$ $\mathrm{m} / \mathrm{z} 263 \cdot 1259$, found $\mathrm{m} / \mathrm{z} 263 \cdot 1252$.

2.1n 5-(2,2-Dimethyl-5-prop-1-ynyl-[1,3]dioxolan4-yl)-pent-1-en-3-ol (32): A solution of ester 31 $(0.54 \mathrm{~g}, 2.25 \mathrm{mmol})$ in toluene $(11 \mathrm{~mL})$ was treated with DIBAL-H $(2.25 \mathrm{~mL}, 1 \mathrm{M}$ solution in toluene) at $-78^{\circ} \mathrm{C}$ in drop wise over a period of $15 \mathrm{~min}$. After being stirred at $-78^{\circ} \mathrm{C}$ for $30 \mathrm{~min}$ the reaction mixture was treated with $\mathrm{MeOH}$ and then allowed to warm to room temperature. Saturated solution of potassium sodium L-tartrate tetrahydrate was added, stirred for $30 \mathrm{~min}$ and extracted with ethyl acetate. The organic layer was washed with brine, dried $\left(\mathrm{Na}_{2} \mathrm{SO}_{4}\right)$, concentrated and the crude product was used for further reaction without any purification. $R_{f}=0.43 \quad(2: 8$ ethyl acetate/hexanes); IR (neat) $3019,2933,2730,1723,1381,1372 \mathrm{~cm}^{-1}$.

To a stirred solution of above aldehyde $(0.44 \mathrm{~g}$, $2.25 \mathrm{mmol})$ in THF $(11 \mathrm{~mL})$ was added vinyl magnesium bromide $(4.5 \mathrm{~mL}, 1 \mathrm{M}$ solution in THF) drop wise at $-78^{\circ} \mathrm{C}$, stirred for $1 \mathrm{~h}$ at $-78^{\circ} \mathrm{C}$ and then for $12 \mathrm{~h}$ at room temperature. The reaction mixture was quenched with saturated $\mathrm{NH}_{4} \mathrm{Cl}$ solution, extracted with ethyl acetate. The organic layer was washed with water, brine, dried $\left(\mathrm{Na}_{2} \mathrm{SO}_{4}\right)$, concentrated and purified by silica gel chromatography (20\% EtOAc in hexanes) to afford the alcohol $32(0.33 \mathrm{~g})$ as a colourless oil in $66 \%$ yield for two steps. $R_{f}=0.48$ (3: 7 ethyl acetate/hexanes); $[\alpha]_{D}^{25}=-29.92$ (c 1.27, $\mathrm{CHCl}_{3}$ ); IR (neat) 3446, 3016, 2253, $1381 \mathrm{~cm}^{-1} ;{ }^{1} \mathrm{H}$ NMR $\left(300 \mathrm{MHz}, \mathrm{CDCl}_{3}\right): \delta$ 5.95-5.83 $(m, 1 \mathrm{H}$, $\mathrm{CH}=), 5.26(d d d, 1 \mathrm{H}, J=17 \cdot 1,3 \cdot 3,1.5 \mathrm{~Hz}, \mathrm{CH}$ of $\left.\mathrm{CH}_{2}=\right), 5.13(d d d, 1 \mathrm{H}, J=10.2,1.5,1.5 \mathrm{~Hz}, \mathrm{CH}$ of $\left.\mathrm{CH}_{2}=\right)$, 4.76-4.71 (m, 1 H, CHO), 4.22-4.11 (m, $1 \mathrm{H}, \mathrm{C} \underline{\mathrm{H}}), 4.09-4.03(m, 1 \mathrm{H}, \mathrm{C} \underline{\mathrm{H}}), 1.87(d, J=2.1 \mathrm{~Hz}$, $\left.3 \mathrm{H}, \underline{\mathrm{CH}}_{3}\right), 1.52\left(s, 3 \mathrm{H}, \mathrm{C}_{3}\right), 1.34\left(s, 3 \mathrm{H}, \underline{\mathrm{CH}}_{3}\right)$; ${ }^{13} \mathrm{C}$ NMR $\left(75 \mathrm{MHz}, \mathrm{CDCl}_{3}\right): \delta 140 \cdot 8,114.8,109 \cdot 4$, $83 \cdot 3,81 \cdot 4,75 \cdot 4,72 \cdot 5,70 \cdot 9,33 \cdot 2,28 \cdot 02,27 \cdot 7,27 \cdot 1$, 26.5, 3.8; HRMS (ES) calcd. for $\mathrm{C}_{13} \mathrm{H}_{20} \mathrm{O}_{3} \mathrm{Na} \mathrm{m} / z$ $247 \cdot 1310$, found $m / z 247 \cdot 1315$.

2.10 5-(2,2-Dimethyl-5-prop-1-ynyl-[1,3]dioxolan4-yl)-pent-1-en-3-one (19): A solution of allylic alcohol $32(0.2 \mathrm{~g}, 0.89 \mathrm{mmol})$ in $\mathrm{CH}_{2} \mathrm{Cl}_{2}(20 \mathrm{~mL})$ was treated with $\mathrm{MnO}_{2}(1.55 \mathrm{~g}, 17.9 \mathrm{mmol})$ at room temperature and the stirring was continued for $12 \mathrm{~h}$. The reaction mixture was filtered through Celite and concentrated. The crude product was purified by silica gel chromatography $(8: 22$ ethyl acetate/hexanes) to afford the ketone $19(0.14 \mathrm{~g})$ as a colourless oil in $70 \%$ yield. $R_{f}=0.6 \quad(20 \%$ EtOAc in hexanes); $[\alpha]^{20}{ }_{\mathrm{D}}=-17.577\left(c 0.355, \mathrm{CHCl}_{3}\right)$; IR (neat) 2988, 2925, 2250, 1703, 1684, $1238 \mathrm{~cm}^{-1}$; ${ }^{1} \mathrm{H}$ NMR $\left(400 \mathrm{MHz}, \mathrm{CDCl}_{3}\right): \delta 6.41-6.24(\mathrm{~m}, 1 \mathrm{H}, \mathrm{C} \underline{\mathrm{H}}=), 6 \cdot 35$ $(d, 1 \mathrm{H}, J=10.4 \mathrm{~Hz}, \mathrm{C} \underline{\mathrm{H}}=), 5.86(d, 1 \mathrm{H}, J=10.4 \mathrm{~Hz}$, C$=), 4.21(d, 1 \mathrm{H}, J=8 \mathrm{~Hz}, \underline{\mathrm{CH}}), 3.95(d t, 1 \mathrm{H}$, 
$J=8,6 \mathrm{~Hz}, \mathrm{C} \underline{\mathrm{H}}), 2 \cdot 87-2.7\left(m, 2 \mathrm{H}, \mathrm{C}_{2}\right), 2.08-1.90$ $\left(m, 1 \mathrm{H}, \mathrm{CH}\right.$ of $\left.\mathrm{CH}_{2}\right), 1.86\left(d, 3 \mathrm{H}, J=1.2 \mathrm{~Hz}, \mathrm{CH}_{3}\right)$, $1.88-1.73\left(m, 1 \mathrm{H}, \mathrm{C} \underline{\mathrm{H}}\right.$ of $\left.\mathrm{C}_{2}\right), 1.45\left(\mathrm{~s}, 3 \mathrm{H}, \mathrm{CH}_{3}\right)$, $1.39\left(s, 3 \mathrm{H}, \mathrm{CH}_{3}\right) ;{ }^{13} \mathrm{C}$ NMR $\left(100 \mathrm{MHz}, \mathrm{CDCl}_{3}\right): \delta$ $200 \cdot 1,136 \cdot 5,128 \cdot 4,109 \cdot 6,83 \cdot 6,80 \cdot 7,70 \cdot 9,70 \cdot 3,35 \cdot 6$, $27 \cdot 2,26 \cdot 6,25 \cdot 9,3 \cdot 8$; HRMS (ES) calcd. for $\mathrm{C}_{13} \mathrm{H}_{18} \mathrm{O}_{3} \mathrm{Na} m / z$ 245.1154, found $\mathrm{m} / z$ 245.1148.

2.1p 5-[2,2-Dimethyl-5-(2-methyl-1-methylene-allyl)[1,3]dioxo-lan-4-yl]-pent-1-en-3-one (33): A solution of enynone $19(0.047 \mathrm{~g}, 0.21 \mathrm{mmol})$ in toluene $(8 \mathrm{~mL})$ was purged with ethylene for $20 \mathrm{~min}$. A solution of Grubbs' catalyst $17(0.018 \mathrm{~g}, 0.021 \mathrm{mmol})$ in toluene $(1 \mathrm{~mL})$ was added and the stirring was continued at room temperature for $36 \mathrm{~h}$ under an atmosphere of ethylene. Then, dimethyl sulphoxide $(0.16 \mathrm{~mL}$, $2.25 \mathrm{mmol}$ ) was added to the reaction mixture and stirred for $12 \mathrm{~h}$ at room temperature. The solvent was evaporated and the crude product was purified by column chromatography $(25 \%$ EtOAc in hexanes) to afford the diene $33(0.027 \mathrm{~g})$ as a yellow oil along with $0.022 \mathrm{~g}$ of unreacted starting material. The yield of the reaction is $86 \%$ based on $53 \%$ conversion. $R_{f}=0.58$ ( $2: 8$ ethyl acetate/hexanes); IR (neat) 2986, 1683,1371, 1241, $1066 \mathrm{~cm}^{-1} ;{ }^{1} \mathrm{H}$ NMR $\left(400 \mathrm{MHz}, \mathrm{CDCl}_{3}\right): \delta 6 \cdot 34-6 \cdot 22(m, 1 \mathrm{H}, \mathrm{CH}=), 6 \cdot 33$ $(d, 1 \mathrm{H}, J=10.4 \mathrm{~Hz}, \mathrm{CH}=), 5.85(d, 1 \mathrm{H}, J=10.4 \mathrm{~Hz}$, $\mathrm{C} \underline{\mathrm{H}}=), 5.41\left(\mathrm{~s}, 1 \mathrm{H}, \mathrm{CH}=\right.$ of $\left.\mathrm{C}_{2}\right), 5.31(s, 1 \mathrm{H}, \mathrm{C} \underline{\mathrm{H}}=$ of $\left.\mathrm{C}_{2}\right), 5.19\left(s, 1 \mathrm{H}, \mathrm{CH}=\right.$ of $\left.\mathrm{C}_{2}\right), 5.05(s, 1 \mathrm{H}$, $\mathrm{C} \underline{\mathrm{H}}=$ of $\left.\mathrm{C}_{2}\right), 4.45(d, 1 \mathrm{H}, J=8.4 \mathrm{~Hz}, \mathrm{C} \underline{\mathrm{H}}), 3.8(d t$, $1 \mathrm{H}, J=8.4,6 \mathrm{~Hz}, \mathrm{CH}), 2.9-2.7\left(m, 2 \mathrm{H}, \mathrm{CH}_{2}\right)$, 2.04-1.97 (m, 1 H, CH of $\left.\mathrm{CH}_{2}\right), 1.94\left(s, 3 \mathrm{H}, \mathrm{CH}_{3}\right)$, $1.82-1.71\left(m, 1 \mathrm{H}, \mathrm{CH}\right.$ of $\left.\mathrm{CH}_{2}\right), 1.46\left(s, 3 \mathrm{H}, \mathrm{CH}_{3}\right)$, $1.44\left(s, 3 \mathrm{H}, \mathrm{CH}_{3}\right) ;{ }^{13} \mathrm{C}$ NMR $\left(100 \mathrm{MHz}, \mathrm{CDCl}_{3}\right): \delta$ $200 \cdot 2,144 \cdot 9,141 \cdot 7,136 \cdot 7,128 \cdot 4,114 \cdot 4,114 \cdot 1$, $108 \cdot 5,80 \cdot 9,80 \cdot 7,36 \cdot 2,27 \cdot 5,27 \cdot 2,26 \cdot 8,22 \cdot 3 \cdot$; HRMS (ES) calcd. for $\mathrm{C}_{15} \mathrm{H}_{22} \mathrm{O}_{3} \mathrm{Na} m / z$ 273.1467, found $\mathrm{m} / z 273 \cdot 1462$.

2.1q 4,4,13-Trimethyl-3,5,-dioxa-tricyclo[8.3.1.0 $\left.0^{2,6}\right]$ tetradec-1(13)-en-9-one (18): A solution of enyne $19(0.1 \mathrm{~g}, 0.45 \mathrm{mmol})$ in toluene $(18 \mathrm{~mL})$ was purged with ethylene for $20 \mathrm{~min}$. A solution of Grubbs' catalyst $17(0.038 \mathrm{~g}, 0.045 \mathrm{mmol})$ in toluene $(1 \mathrm{~mL})$ was added and then heated at $80^{\circ} \mathrm{C}$ for $48 \mathrm{~h}$ under an atmosphere of ethylene. Then, dimethyl sulphoxide $(0.16 \mathrm{~mL}, 2.25 \mathrm{mmol})$ was added to the reaction mixture and stirred for $12 \mathrm{~h}$ at room temperature. The solvent was evaporated and the crude product was purified by column chromatography $(25 \%$ EtOAc in hexanes) to afford the diene $18(0.07 \mathrm{~g})$ as a white solid in $62 \%$ yield. $R_{f}=0.44$ (2:8 ethyl acetate/ hexanes); m.p. $=98-100^{\circ} \mathrm{C} ;[\alpha]^{25}{ }_{\mathrm{D}}=-14.52(c 0.57$, $\mathrm{CHCl}_{3}$ ); IR (KBr) 3352, 2921, 1701, $1445 \mathrm{~cm}^{-1} ;{ }^{1} \mathrm{H}$ NMR $\left(400 \mathrm{MHz}, \mathrm{CDCl}_{3}\right): \delta 4 \cdot 14-4.05(m, 2 \mathrm{H}$, $2 \times \underline{\mathrm{CH}}$ of $\left.\mathrm{CH}_{2}\right), 2.85\left(d, 2 \mathrm{H}, J=10.4 \mathrm{~Hz}, \underline{\mathrm{CH}}_{2}\right), 2.6$ $(d t, 1 \mathrm{H}, J=11 \cdot 6,3 \cdot 2 \mathrm{~Hz}, \mathrm{C} \underline{\mathrm{H}}), 2 \cdot 3-1.88(m, 6 \mathrm{H}$, $\left.3 \times \underline{\mathrm{CH}}_{2}\right), 1.81\left(\mathrm{~s}, 3 \mathrm{H}, \mathrm{C}_{3}\right), 1.71-1.62\left(\mathrm{~m}, 2 \mathrm{H}, \underline{\mathrm{CH}}_{2}\right)$, $1.45\left(s, 3 \mathrm{H}, \mathrm{CH}_{3}\right), 1.44\left(s, 3 \mathrm{H}, \mathrm{CH}_{3}\right) ;{ }^{13} \mathrm{C} \mathrm{NMR}$ $\left(75 \mathrm{MHz}, \mathrm{CDCl}_{3}\right): \delta 213 \cdot 2,145 \cdot 5,123.5,107 \cdot 9$, $78 \cdot 8,78 \cdot 3,49 \cdot 4,36 \cdot 7,29 \cdot 1,27 \cdot 4,27 \cdot 1,27 \cdot 03,26 \cdot 4$, 19.3, 18.9; LRMS (ES) $[\mathrm{M}+\mathrm{Na}]^{+} 273 \cdot 2426$; HRMS (ES) calcd. for $\mathrm{C}_{15} \mathrm{H}_{22} \mathrm{O}_{3} \mathrm{Na} \mathrm{m} / z$ 273.1467, found $\mathrm{m} / z$ $273 \cdot 1475$.

\section{Results and discussion}

In connection to this issue of generating simpler analogues of taxol in relatively fewer steps and also in continuation of our interest in synthesis of complex natural products with significant biological activity, we began a research program to develop a simple strategy to achieve this objective. We believed that the best way of reducing the number of steps would be utilization of domino reactions, which are considered to be superior to step-wise procedures as several reactions can be combined in a single step, and consequently the synthesis can be shortened significantly. ${ }^{10}$ Furthermore, domino reactions avoid the unnecessary isolations and purifications of intermediates in multistep transformations and reduce considerable amount of solvents required for the purification, which is hazardous to environment, especially, in bulk scale preparations. Among domino reactions, the domino enyne metathesis/intramolecular Diels-Alder reaction is particularly attractive because of its elegance in generating bicyclic ring system with defined stereochemistry. ${ }^{11}$ As a part of our chiron approach ${ }^{12}$ towards the synthesis of simpler analogues of biologically active natural products from carbohydrates, we have already described a synthetic route for the AB-ring of taxol without gem dimethyl group employing the cross enyne metathesis/intramolecular Diels-Alder reaction (IMDAR) ${ }^{12 b}$ and here, we present the full account of all our attempts directed toward the generation of functionalized taxol rings. From a retro-synthetic perspective (scheme 1), we envisaged that the 6, 8-fused bicyclic compound $\mathbf{6}$, corresponding to $\mathrm{BC}$-ring of taxol, could be obtained by opening the furanoside ring of ketal 7 which in turn could be obtained from the corresponding enyne 8 by a domino intramolecular 

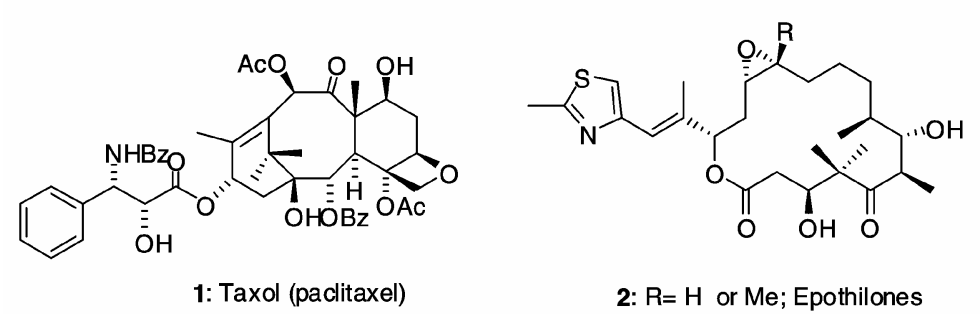

2: $\mathrm{R}=\mathrm{H}$ or Me; Epothilones
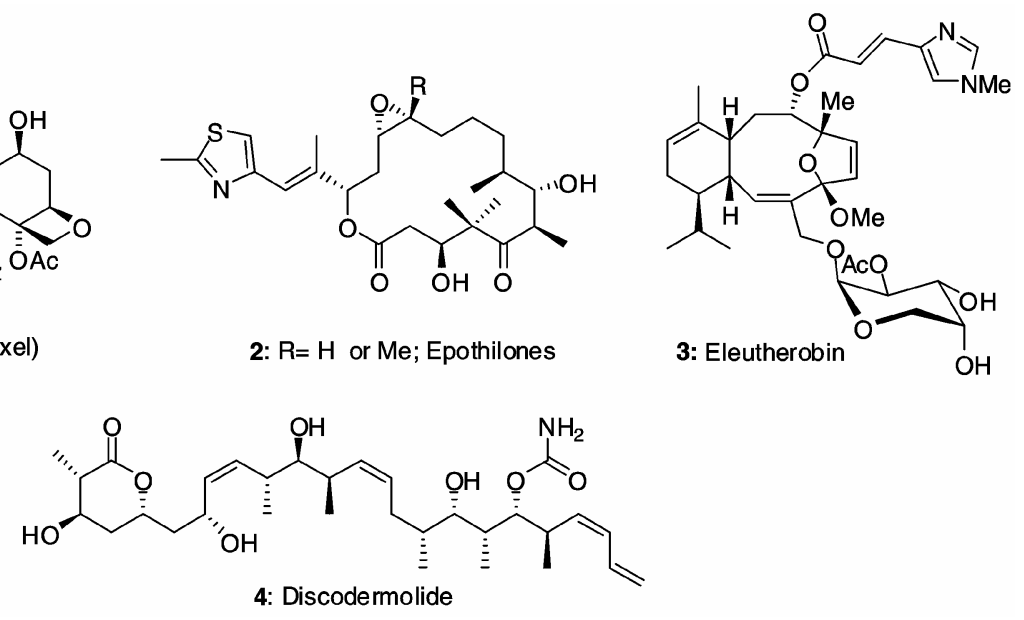

Figure 1. Microtubules stabilizing cytotoxic agents.

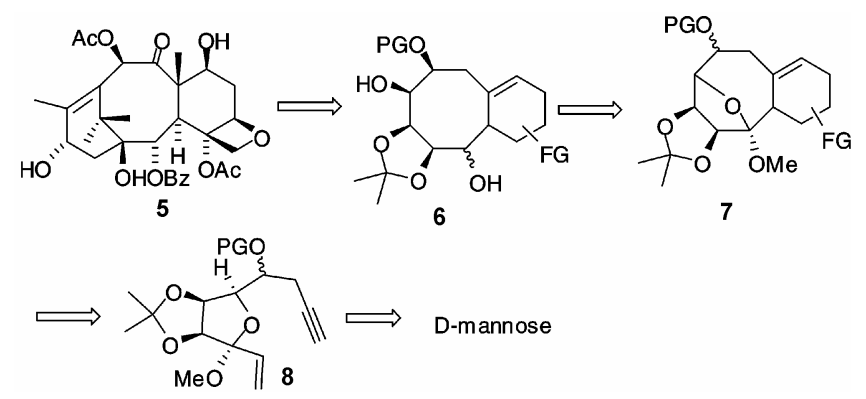

Scheme 1.

enyne metathesis/intermolecular Diels-Alder reaction and the requisite enyne 8 could be obtained from $\mathrm{D}-(+)$-mannose through a sequence of reactions.

Accordingly, our synthesis (scheme 2) towards the highly oxygenated BC-ring of taxol 6 began with the D-(+)-mannose diacetonide 9 which was converted to the diol 10 using the protocol developed in our laboratory ${ }^{13}$ and then, diol 10 was converted to aldehyde 11 using silica supported sodium periodide. ${ }^{14}$ Treatment of aldehyde $\mathbf{1 1}$ under a Barbier type reaction ${ }^{15}$ with propargyl bromide in the presence of zinc led to a diasteroemetic mixture alcohol 12 in $30: 1$ ratio as confirmed by ${ }^{1} \mathrm{H}$ NMR spectroscopy. Alcohol $\mathbf{1 2}$ was subsequently protected as its TBS ether to furnish 13. Having the enyne in hand, we attempted the key intramolecular enyne metathesis reaction ${ }^{16}$ to generate the B ring of taxol as a 1,3-diene which in situ could be reacted with appropriate dienophiles to afford the corresponding Diels-Alder adducts.

Unfortunately, our preliminary attempts with Grubbs' I generation catalyst $\mathbf{1 6}$ as well as more reactive Grubbs' II generation catalyst $\mathbf{1 7}$ in refluxing
$\mathrm{CH}_{2} \mathrm{Cl}_{2}$ under an argon atmosphere did not lead to the desired product though starting material could be recovered. Nevertheless, when the enyne metathetic reaction was attempted with catalyst $\mathbf{1 7}$ in ethylene atmosphere, the cross enyne metathesis product 14 was obtained as a result of the reaction between the alkyne part and the ethylene gas which suggested that the alkene part is too inert to afford the intramolecular enyne metathesis product. Consequently, our further attempts of ring closing metathesis on $\mathbf{1 4}$ using the catalyst 17 were also not fruitful.

Although our attempts to generate the BC-ring of taxol using domino enyne metathesis/DAR reaction were not successful, it offered an essential clue that the intermolecular enyne metathesis reaction between ethylene and an alkyne could be employed as a key reaction in our synthesis. ${ }^{17}$ Toward this end, we have devised a strategy based on domino cross enyne metathesis/intramolecular DAR for the synthesis of bicyclo[5.3.1] undecene as described in scheme 3. It is noteworthy that, bicyclo[5.3.1] undecene is an integral part of taxol and it corresponds to the AB-ring of the molecule without the gem dimethyl group. ${ }^{18}$ Regardless of several excellent contributions from various groups for the synthesis of the $\mathrm{AB}$-ring of taxol via an intramolecular Diels-Alder reaction (IMDAR) ${ }^{19}$ the domino enyne cross-metathesis/ IMDAR has not been explored, prior to our preliminary communication, ${ }^{12 b}$ for the construction of the corresponding 10 -membered ring bridged by one carbon, which is bicyclo[5.3.1] undecene.

We envisaged that a domino enyne cross-metathesis/IMDAR could be an ideal key step for the construction of a bicyclo[5.3.1] undecene corresponding to the AB-ring of taxol but without the 

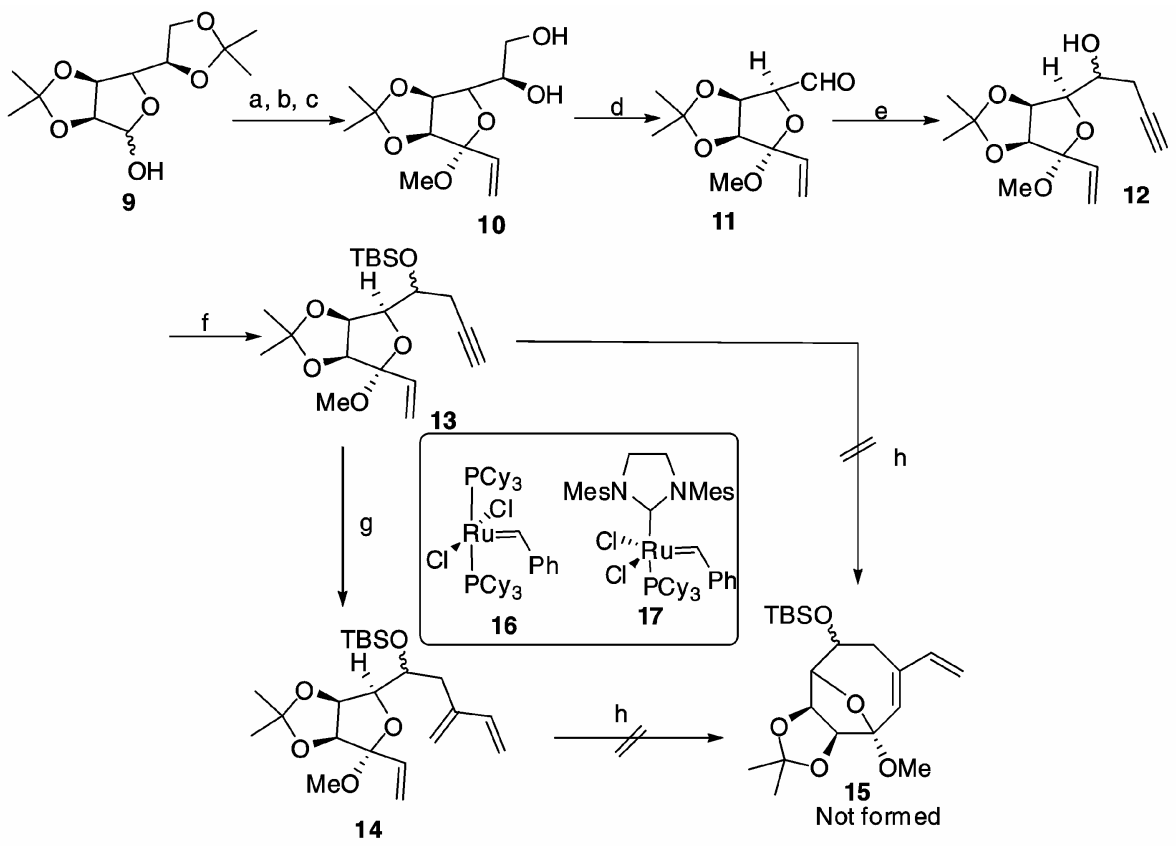

Scheme 2. Reagents and conditions: (a) $\mathrm{CH}_{2}=\mathrm{CHMgBr}, \mathrm{THF}, 0^{\circ} \mathrm{C}$ to r.t., $12 \mathrm{~h}$, $90 \%$; (b) $\mathrm{MnO}_{2}, \mathrm{CH}_{2} \mathrm{Cl}_{2}$, r.t., 6 h, 92\%; (c) PPTS, $\mathrm{MeOH}$, r.t., 12 h, 88\%; (d) $\mathrm{NaIO}_{4}$, silica, $\mathrm{CH}_{2} \mathrm{Cl}_{2}$, r.t., $2 \mathrm{~h}$; (e) propargyl bromide, $\mathrm{Zn}$, THF, r.t., $3 \mathrm{~h}, 67 \%$ over two steps; (f) $\mathrm{TBSCl}, \mathrm{Im}, \mathrm{DMF}, 50^{\circ} \mathrm{C}, 24 \mathrm{~h}, 83 \%$; (g) 17, ethylene, $\mathrm{CH}_{2} \mathrm{Cl}_{2}$, r.t., $3 \mathrm{~h}$, $64 \%$; (h) $17, \mathrm{CH}_{2} \mathrm{Cl}_{2}$, reflux.<smiles>C=CC#CC1OC(C)(C)O[C@@H]1CCC(=O)C=C</smiles>

Scheme 3.

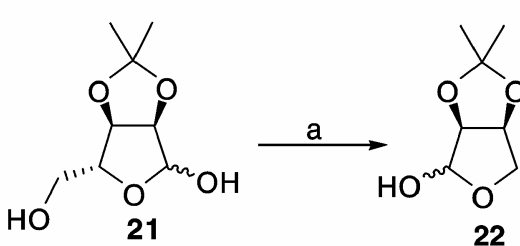<smiles>COP(=O)(OC)C(C)=O</smiles><smiles>C#C[C@H]1OC(C)(C)O[C@H]1CO</smiles><smiles>CC1(C)O[C@H]2C#C[13CH]C[C@H]2OC1(C)C</smiles>

25

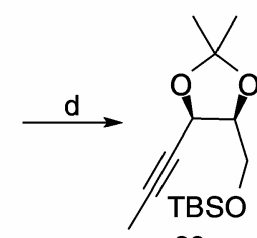

26

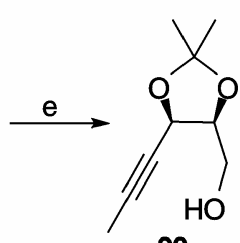

20

Scheme 4. Reagents and conditions: (a) $\mathrm{NaBH}_{4}, \mathrm{MeOH}, 0^{\circ} \mathrm{C}, 2 \mathrm{~h}$; then $\mathrm{NaIO}_{4}$, $\mathrm{H}_{2} \mathrm{O}, 3 \mathrm{~h}, 86 \%$; (b) $\mathrm{K}_{2} \mathrm{CO}_{3}, \mathrm{MeOH}$, reflux, $6 \mathrm{~h}, 76 \%$ (c) TBSCl, Im, cat. DMAP, $\mathrm{CH}_{2} \mathrm{Cl}_{2}$, r.t., 2 h, $78 \%$; (d) ${ }^{n} \mathrm{BuLi}, \mathrm{CH}_{3} \mathrm{I}, \mathrm{HMPA}, \mathrm{THF},-78^{\circ} \mathrm{C}$ to r.t., 12 h, $88 \%$; (e) TBAF, THF, r.t., 2 h, $91 \%$. 
gem dimethyl group. From a retrosynthetic perspective (scheme 3), we considered the construction of 18 via domino cross-enyne metathesis/IMDA reaction of enyne 19. This acyclic ketone 19 could, in turn, be obtained from the alkynol 20. The alcohol 20 could then be derived from D-(+)-ribose monoacetonide $\mathbf{2 1}$ in a few steps. Our synthetic sequence (scheme 4) started with the known lactol $\mathbf{2 2},{ }^{20}$ which was smoothly converted into the alkyne 24 by following the Ohira-Bestmann protocol in refluxing methanol in $76 \%$ yield. ${ }^{21}$ The primary alcohol $\mathbf{2 4}$ was then protected as its TBS ether $\mathbf{2 5}$ before treatment with ${ }^{n} \mathrm{BuLi}$ and MeI to afford 26 in excellent yield. Removal of the TBS group was then easily achieved with TBAF to afford the alcohol 20 in $91 \%$ yield.

After the successful synthesis of alcohol 20, our next goal was to extend two more carbons on the right hand side of this molecule to afford 19 . We envisaged that the alcohol $\mathbf{2 0}$ could be converted to the corresponding iodo compound $\mathbf{2 7}$, which on reaction with the ethylacetoacetate in the presence of base should afford 28 (scheme 5). Decarboxylation followed by a Mannich reaction ${ }^{22}$ of 28 should provide 19 , the precursor for the domino reaction in relatively less steps. Towards this end, the alcohol 20 was converted to the corresponding tosylate which on reaction with $\mathrm{NaI}$ in refluxing ethylmethyl ketone afforded the iodo compound 27. But unfortunately, our attempts to synthesize $\mathbf{2 8}$ from $\mathbf{2 7}$ were unsuccessful under a variety of reaction conditions utilizing different bases, such as $\mathrm{NaH}$, NaOEt and $\mathrm{KO}^{t} \mathrm{Bu}$.

Puzzled with the failure of the alkylation reaction, we decided to replace ethylacetoacetate with acetonitrile and effect the alkylation using LDA. Accordingly, the iodo compound $\mathbf{2 7}$ was treated with acetonitrile in the presence of $\mathrm{LDA}^{23 a}$ to afford the nitrile 29 in $11 \%$ yield along with unreacted starting material. When excess of LDA was used to improve the conversion of product, dialkylation product was observed as the predominant product. On the other hand, replacement of LDA with ${ }^{n} \mathrm{BuLi}$ improved the yield of reaction to $21 \%$ along with unreacted starting material. ${ }^{23 b}$ However, the subsequent Grignard reaction which would have furnished the ketone $\mathbf{1 9}$ in a single step was unsuccessful despite our repeated attempts and the starting material was recovered as unchanged.

In an effort to find an alternative route, we modified our strategy to synthesize 19 as delineated in the scheme 6 . We anticipated that the alcohol could be converted to $\alpha, \beta$-unsaturated ester 30, which in turn could be reduced to give the saturated ester $\mathbf{3 1}$ thereby providing the desired extension of two carbon atoms. Accordingly, we started this approach with the oxidation of alcohol 20, under Swern conditions to provide an aldehyde, which was subsequently subjected to Wittig reaction to afford $\mathbf{3 0}$ in $60 \%$ yield over two steps (scheme 6). Our next task was to reduce the double bond of enone $\mathbf{3 0}$ selectively in the presence of the alkyne. Unfortunately, conventional reduction methods such as $\mathrm{Mg} /$ $\mathrm{MeOH},{ }^{24} \mathrm{NiCl}_{2} / \mathrm{NaBH}_{4},{ }^{25}$ copper(I) hydride cluster $\left[\left(\mathrm{Ph}_{3} \mathrm{P}\right) \mathrm{CuH}\right]_{6}{ }^{26}$ and $\mathrm{CuI} / \mathrm{LAH}^{27}$ did not yield the desired product. Finally, we were relieved to find that $\mathrm{Cu}_{2} \mathrm{Cl}_{2}$ ( 0.75 equiv)/ $\mathrm{NaBH}_{4}$ (6 equiv) effectively re-

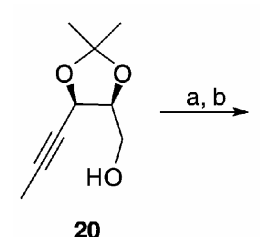

20

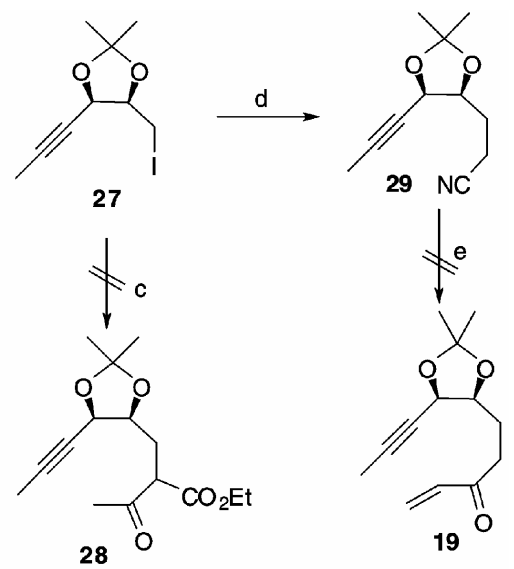

Scheme 5. Reagents and conditions: (a) $\mathrm{TsCl}, \mathrm{Py}$, cat. DMAP, 12 h, 87\%; (b) NaI, EMK, reflux, 12 h, 83\%; (c) ethylacetoacetate, base; (d) $\mathrm{CH}_{3} \mathrm{CN},{ }^{n} \mathrm{BuLi}, \mathrm{THF},-78^{\circ} \mathrm{C}$, $1.5 \mathrm{~h}, 21 \%$; (e) $\mathrm{CH}_{2}=\mathrm{CHMgBr}$, ether, $-78^{\circ} \mathrm{C}$ to r.t., $12 \mathrm{~h}$.

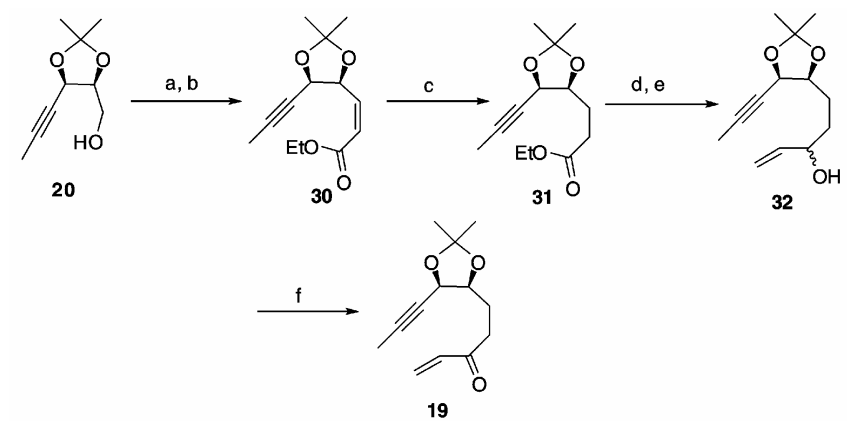

Scheme 6. Reagents and conditions: (a) $(\mathrm{COCl})_{2}$, DMSO, $\mathrm{Et}_{3} \mathrm{~N}, \mathrm{CH}_{2} \mathrm{Cl}_{2},-78^{\circ} \mathrm{C}, 1 \mathrm{~h}$; (b) carboethoxymethylenetriphenylphosph-orrane, $\mathrm{CH}_{3} \mathrm{CN}, \quad$ r.t., $12 \mathrm{~h}$, $68 \%$; (c) $\mathrm{Cu}_{2} \mathrm{Cl}_{2}, \mathrm{NaBH}_{4}, \mathrm{THF}, \mathrm{MeOH},-20^{\circ} \mathrm{C}, 30 \mathrm{~min}$, $80 \%$; (d) DIBAL-H, toluene, $-78^{\circ} \mathrm{C}, 30 \mathrm{~min}, 84 \%$; (e) $\mathrm{CH}_{2}=\mathrm{CHMgBr}$, THF, $-78^{\circ} \mathrm{C}$ to r.t., $12 \mathrm{~h}, 53 \%$; (f) $\mathrm{MnO}_{2}$, $\mathrm{CH}_{2} \mathrm{Cl}_{2}$, r.t., $12 \mathrm{~h}, 67 \%$. 

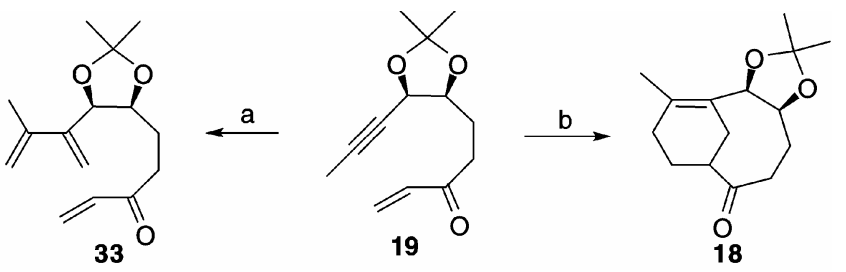

Scheme 7. Reagents and conditions: (a) 17 (10 mol\%), ethylene, toluene, r.t., $36 \mathrm{~h}, 86 \%$ based on $53 \%$ conversion; (b) 17 (10 mol\%), ethylene, toluene, $80^{\circ} \mathrm{C}, 24 \mathrm{~h}$, $62 \%$.

duced this unsaturated ester selectively at $-20^{\circ} \mathrm{C}$ to provide 31 in $80 \%$ yield. ${ }^{28}$ Subsequently, 31 was treated with DIBAL-H to afford the aldehyde, which on treatment with vinyl magnesium bromide afforded a diastereomeric mixture of allylic alcohols 32 in $66 \%$ yield for two steps. The oxidation of allylic alcohol 32 with $\mathrm{MnO}_{2}$ afforded the ketone 19 in $67 \%$ yield. The alkenynone 19 was found to be very unstable and polymerized quite rapidly even in the refrigerator.

Synthesis of 19 set the stage for the domino cross enyne metathesis/IMDA reaction. When we carried out this reaction under $1 \mathrm{~atm}$. of ethylene in the presence of $\mathbf{1 7}$ in toluene at room temperature for $36 \mathrm{~h}$ (scheme 7), we isolated the trienone 33 in $86 \%$ yield based on $53 \%$ conversion. ${ }^{29}$ We were hopeful that a domino cross enyne/IMDA reaction could be achieved if we performed the reaction at higher temperature, and indeed at $80^{\circ} \mathrm{C}$, we were delighted to see a smooth domino enyne metathesis/ IMDA reaction of enyne 19 to afford a single diastereomer of the bicyclo[5.3.1] undecanone $\mathbf{1 8}$ which corresponds to the AB-ring skeleton of taxol without the gem dimethyl group in $62 \%$ yield.

\section{Conclusions}

In conclusion, our preliminary efforts to generate the $\mathrm{BC}$-ring of taxol using enyne metathesis/intermolecular DAR led to the formation of only a triene which was formed by an intermolecular enyne metathesis with ethylene. However, we could successfully develop a simple and straightforward tandem enyne crossmetathesis/IMDA reaction strategy for the construction of bicyclo[5.3.1] undecene system which is the structural sub-unit that constitutes AB-ring system of taxol without the gem dimethyl group. Efforts are underway in our laboratory to extend this strategy to synthesize the core structure and finally to synthesize taxol.

\section{Acknowledgements}

Authors acknowledge Department of Science and Technology (DST), New Delhi for financial support and SAIF, IIT Bombay for providing spectral facilities. VRK thanks Council of Scientific and Industrial Research (CSIR), New Delhi for a fellowship.

\section{References}

1. Wani M C, Taylor H L, Wall M E, Coggon P and McPhail A T 1971 J. Am. Chem. Soc. 932325

2. For review on taxol, see: Kingston D G I 2001 Chem. Commun. 867

3. (a) Holton R A, Somoza C, Kim H B, Liang F, Biediger R J, Boatman P D, Shindo M, Smith C C, Kim S, Nadizadeh H, Suzuki Y, Tao C, Vu P, Tang S, Zhang P, Murthi K K, Gentile L N and Liu J H 1994 J. Am. Chem. Soc. 116 1597; (b) Holton R Somoza A C, Kim H B, Liang F, Biediger R J, Boatman P D, Shindo M, Smith C C, Kim S, Nadizadeh H, Suzuki Y, Tao C, Vu P, Tang S, Zhang P, Murthi K K, Gentile L N and Liu J H 1994 J. Am. Chem. Soc. 116 1599

4. (a) Nicolaou K C, Nantermet P G, Ueno H, Guy R K, Couladouros E A and Sorensen E J $1995 \mathrm{~J}$. Am. Chem. Soc. 117 624; (b) Nicolaou K C, Liu J-J, Yang Z, Ueno H, Sorensen E J, Claiborne C F, Guy R K, Hwang C-K, Nakada M and Sorensen E J $1995 \mathrm{~J}$. Am. Chem. Soc. 117 634; (c) Nicolaou K C, Yang Z, Liu J-J, Nantermet P G, Claiborne C F, Renaud J, Guy R $\mathrm{K}$ and Shibayama K $1995 \mathrm{~J}$. Am. Chem. Soc. 117 645; (d) Nicolaou K C, Ueno H, Liu J-J, Nantermet P G, Yang Z, Renaud J, Paulvannan K and Chadha R J 1995 J. Am. Chem. Soc. 117653

5. Danishefsky S J, Masters J J, Young W B, Link J T, Snyder L B, Magee T V, Jung D K, Isaacs R C A, Bornmann W G, Alaimo C A, Coburn C A and DiGrandi M J 1996 J. Am. Chem. Soc. 1182843

6. (a) Wender P A, Badham N F, Conway S P, Floreancig $\mathrm{P}$ E, Glass T E, Granicher C, Houze J B, Janichen J, Lee D, Marquess D G, McGrane P L, Meng W, Mucciaro T P, Muhlebach M, Natchus M G, Paulsen H, Rawlins D B, Satkofsky J, Shuker A J, Sutton J C, Taylor R E and Tommoka K $1997 \mathrm{~J}$. Am. Chem. Soc. 119 2755; (b) Wender P A, Badham N F, Conway S P, Floreancig P E, Glass T E, Houze J B, Krauss N E, Lee D, Marquess D G, McGrane P L, Meng W, Natchus M G, Shuker A J, Sutton J C and Taylor R E 1997 J. Am. Chem. Soc. 1192757

7. (a) Hara R, Kawahara S, Nishimori T, Kashima H, Nakamura N, Morihira K and Kuwajima I $1998 J$. Am. Chem. Soc. 120 12980; (b) Kusama H, Hara R, Kawahara S, Nishimori T, Kashima H, Nakamura N, Morihira K and Kuwajima I $2000 \mathrm{~J}$. Am. Chem. Soc. 1223811

8. Mukaiyama $T$, Shiina I, Iwadare $H$, Saitoh $M$, Nishimura T, Ohkawa N, Sakoh H, Nishimura K, Tani Y I, Hasegawa M, Yamada K and Saitoh K 1999 Chem. Eur. J. 5121 
9. Holton R A, Biediger R J and Boatman P D 1995 In Taxol®: Science and Applications (ed.) M Suffness (FL: CRC Press) pp 97-122

10. For reviews on domino reactions, see: (a) Posner G H 1986 Chem Rev. 86 831; (b) Ho T-L 1992 Tandem organic reactions (New York: Wiley); (c) Tietze L F and Beifuss U 1993 Angew. Chem., Int. Ed. Engl. 32 131; (d) Fukumoto K 1994 J. Synth. Org. Chem. Jpn. 52 2; (e) Bunce R A 1995 Tetrahedron 51 13103; (f) Tietze L F 1996 Chem Rev. 96 115; (g) Parsons P J, Penkett C S and Shell A J 1996 Chem. Rev. 96 195; (h) Tietze L F and Rackelmann N 2004 Pure Appl. Chem. 76 1967; (i) Pellissier H 2006 Tetrahedron 62 1619; (j) Tietze L F, Brasche G and Gericke K M 1992 Domino reactions in organic synthesis (Wiley$\mathrm{VCH})$

11. (a) Schurer S C and Blechert S 1999 Tetrahedron Lett. 40 1877; (b) Schurer S C and Blechert S 1999 Chem. Commun. 1203; (c) Kotha S, Halder S and Brahmachari E 2002 Tetrahedron 589203

12. (a) Kaliappan K P and Ravikumar V 2005 Org. Biomol. Chem. 3 848; (b) Kaliappan K P and Ravikumar V and Pujari S A 2006 Tetrahedron Lett. 47981

13. Kaliappan K P, Das P and Kumar N 2005 Tetrahedron Lett. 463037

14. Zhong Y-L and Shing T K M 1997 J. Org. Chem. 62 2622

15. Pakulski S and Zamojsky A 1997 Tetrahedron 53 2653

16. For reviews on enyne metathesis, see: (a) Mori M 1998 In Enyne metathesis in Top. Organomet. Chem. (ed.) A Furstner (Berlin Heidelberg: Springer) pp 133; (b) Poulsen C S and Madsen R 2003 Synthesis 1; (c) Diver S T and Giessert A J 2004 Chem. Rev. 104 1317

17. For domino ethylene cross-enyne metathesis/IMDA reaction, see: Nishiguchi N, Kinoshita A and Mori M 2000 Tennen Yuki Kagobutsu Toronkai Koen Yoshishu 42 739, CA No. 134:340567; For domino cross-enyne metathesis/IMDA reaction, see: Mix S and Blechert S 2005 Org. Lett. 72015

18. (a) For review, see: Mehta G and Singh V 1999 Chem. Rev. 99 881; (b) Oh J, Choi J-R and Cha J K 1992 J. Org. Chem. 57 6664; (c) Kraus G A and Zheng D 1993 Synlett 71; (d) Mukaiyama T, Shiina I, Kimura K, Akiyama Y and Iwadare H 1995 Chem. Lett. 229; (e) Shiina I, Iwadare H, Saitoh M, Ohkawa N, Nishimura T and Mukaiyama T 1995 Chem. Lett. 781; (f) Crich D and Natarajan S 1995 Chem. Commun. 85; (g) Banwell M G, Darmos P, McLeod M D and Hockless D C R 1998 Synlett 897; (h) Wenz M, Grobach D, Beitzel M and Blechert S 1999 Synthesis
4 607; (i) Shimada Y, Nakamura M, Suzuka T, Matsui J, Tatsumi T, Tsutsumi K, Morimoto T, Kurosawa H and Kaiuch K 2003 Tetrahedron Lett. 44 14013

19. For an excellent review on IMDA, see: Bear B R, Sparks S M and Shea K J 2001 Angew. Chem., Int. Ed. Engl. 40 820; Also see: (a) Shea K J and Davis $\mathrm{P}$ D 1983 Angew. Chem. Int. Ed. Engl. 22 419; (b) Shea K J and Gilman J 1985 J. Am. Chem. Soc. 107 4791; (c) Bonnert R V and Jenkins P R 1987 J. Chem. Soc. Chem. Commun. 1540; (d) Shea K J and Haffner C D 1988 Tetrahedron Lett. 29 1367; (e) Yadav J S and Ravishankar R 1991 Tetrahedron Lett. 32 2629; (f) Phillips A J, Morris J C and Abell A D 2000 Tetrahedron Lett. 41 2723; (g) Jackson R W and Shea K J 1994 Tetrahedron Lett. 35 1317; (h) Forgione P, Wilson P D, Yap G P A and Fallis A G 2000 Synthesis 921; (i) Winkler J D, Kim S H and Kim H S 1995 Tetrahedron Lett. 36 687; (j) Winkler J D, Kim H S, Kim S H, Ando K and Houk K N 1997 J. Org. Chem. 62 2957; (k) Winkler J D, Holland J M and Peters D A $1996 \mathrm{~J}$. Org. Chem. 61 9074; (1) Vuzquez A and Williams R M $2000 \mathrm{~J}$. Org. Chem. 657865

20. (a) Paquette L A and Bailey S $1995 \mathrm{~J}$. Org. Chem. 60 7849; (b) Krishna U M, Srikanth G S C, Trivedi G K and Deodhar K D 2003 Synlett 2383

21. (a) Ohira S 1989 Synth. Commun. 19 561; (b) Muller S, Liepold B, Roth G J and Bestmann H J 1996 Synlett 521

22. Grast J-L 1978 Tetrahedron Lett. 192955

23. (a) Larcheveque M, Perrior P and Petit Y 1983 Synthesis 297; (b) Taber D F and Kong S 1997 J. Org. Chem. 628575

24. (a) Dominquez C, Csaky A G and Plumet J 1991 Tetrahedron Lett. 32 4183; (b) Youn I K, Yon G H and Pak C S 1986 Tetrahedron Lett. 262409

25. (a) Saksena A K, Lovey R G, Girijavallabhan V M, Ganguly A K and McPhail A T $1986 \mathrm{~J}$. Org. Chem. 51 5024; (b) Stien D and Gastaldi S $2004 \mathrm{~J}$. Org. Chem. 69 4464; (c) Shin Y, Fournier J H, Balachandran R, Madiraju C, Raccor B S, Zhu G, Edler M C, Hamel E, Day B W and Curran D P 2005 Org. Lett. 72873

26. (a) Brestensky D M, Huseland D E, McGettigan C and Stryker J M 1988 Tetrahedron Lett. 29 3749; (b) Mahoney W S, Brestensky D M and Stryker J M $1988 \mathrm{~J}$. Am. Chem. Soc. 110291

27. Ashby E C, Lin J J and Kovar R $1976 \mathrm{~J}$. Org. Chem. 411939

28. Narisada M, Horibe I, Watanabe $F$ and Takeda $K$ 1989 J. Org. Chem. 545308

29. Kinoshita A, Sakakibara N and Mori M 1999 Tetrahedron $\mathbf{5 5} 8155$ 